\title{
Shear-wave Anisotropy in the Earth's Inner Core
}

$3 \quad{ }^{1}$ Research School of Earth Sciences, The Australian National University, ACT 2601, Australia.

5 Corresponding author:

6 Sheng Wang (sheng.wang@anu.edu.au), Hrvoje Tkalčić (hrvoje.tkalcic@anu.edu.au)

8 Key Points:

- We observe shear-wave anisotropy in the Earth's inner-core based on time and amplitude variations of earthquake coda-correlation wavefield

- Inner-core shear waves travel faster for oblique than equatorial angles relative to Earth's rotation axis by at least $\sim 5 \mathrm{~s}(\sim 0.8 \%$ anisotropy $)$

- The new observations rule out one of the bcc-iron models in the inner core, although we cannot uniquely determine the dominating model

16 Keywords:

17 Earth's inner core; Shear-wave anisotropy; Iron crystal structure 


\section{Abstract}

22 Earth's inner core anisotropy is widely used to infer the deep Earth's evolution and present dynamics.

23 Many compressional-wave anisotropy models have been proposed based on seismological observations.

24 In contrast, inner-core shear-wave (J-wave) anisotropy - on a par with the compressional-wave anisotropy

25 - has been elusive. Here we present a new class of the J-wave anisotropy observations utilizing earthquake

26 coda-correlation wavefield. We establish that the coda-correlation feature I2-J, sensitive to J-wave speed,

27 exhibits time and amplitude changes when sampling the inner core differently. J-waves traversing the

28 inner core near its center travel faster for the oblique than equatorial angles relative to the Earth's rotation

29 axis by at least $\sim 5 \mathrm{~s}$. The simplest explanation is the J-wave cylindrical anisotropy with a minimum

30 strength of $\sim 0.8 \%$, formed through the lattice-preferred-orientation mechanism of iron. Although we

31 cannot uniquely determine its stable iron phase, the new observations rule out one of the body-centered-

32 cubic iron models.

\section{Plain Language Summary}

34 Earth's inner core anisotropy - the directional dependence of seismic wave speed in the inner core 35 contains essential information of deep Earth's structure and dynamics. It results from a preferred 36 alignment of iron crystals related to the formation and post-formation dynamics of the inner core. Many 37 studies have investigated the inner core anisotropy observed for compressional waves. In contrast, possible 38 anisotropy for the inner-core shear waves remains elusive. This study presents a new class of inner-core 39 shear-wave anisotropy observations based on recent advances in earthquake coda-correlation wavefield.

40 We find that the coda-correlation feature I2-J, sensitive to the inner-core shear-wave speed, exhibits

41 variable timing and amplitude for sampling the inner core in different directions. Quantitatively, inner42 core shear waves travel faster for at least $\sim 5 \mathrm{~s}$ in directions oblique to the Earth's rotation axis than 43 directions parallel to the equatorial plane. The simplest and most plausible explanation for our 
44 observations is the inner-core shear-wave anisotropy with a strength of $\sim 0.8 \%$ or higher. We can rule out

45 at least one of the body-centered-cubic iron models in the inner core, although the other models are not 46 distinguishable. 


\section{Introduction}

48 It has been eight and a half decades since the discovery of the Earth's inner core (IC) (Lehmann, 1936).

49 Understanding the IC structure and dynamics is of high priority to geoscientists, given its active role in 50 the Earth's evolution (Tkalčić, 2017). This includes coupling with the liquid outer core, which sustains

51 the geodynamo (Braginsky, 1963; Buffett et al., 1996) and possibly affects the lowermost mantle 52 dynamics (Aubert et al., 2008; Gubbins et al., 2011), and even the processes at Earth's surface (Biggin et 53 al., 2015). One direction in IC studies is its elastic anisotropy. The anisotropy strength and volumetric 54 dependence may help decipher the IC evolution and its current state (Tkalčić, 2017). This is because the 55 anisotropic properties reveal a preferred alignment of iron crystals (Stixrude \& Cohen, 1995; Steinle56 Neumann et al., 2001; Belonoshko et al., 2008) formed during the solidification (Karato 1993; Bergman, 57 1997) or post-solidification deformation progress (Jeanloz \& Wenk, 1988; Yoshida et al., 1996; Wenk et 58 al., 2000) coupled with the geodynamo (Karato, 1999; Buffett \& Wenk, 2001). However, it is uncertain 59 which type of iron crystal, hexagonal-close-packed (hcp) (Stixrude \& Cohen, 1995; Steinle-Neumann et 60 al., 2001) or body-centered-cubic (bcc) (Vočadlo et al., 2003; Belonoshko et al., 2008; Calvet \& Margerin, $612008)$ structure, is stabilized in the IC.

63 Pioneering studies characterized the anisotropy based on faster compressional waves traversing the IC in 64 directions quasi-parallel to the Earth's rotation axis than in equatorial directions (Poupinet et al., 1983; 65 Morelli et al., 1986; Woodhouse et al., 1986; see also Shearer et al., 1988; Creager, 1992; Tromp, 1993). 66 However, subsequent observations revealed more complex anisotropic properties of the IC (Figure 1), 67 such as a quasi-isotropic thin layer in the upper IC (e.g., Shearer, 1994; Song \& Helmberger, 1995) and a 68 hemispherical dichotomy of the IC (e.g., Tanaka \& Hamaguchi, 1997; Niu \& Wen, 2001; Waszek \& Deuss, 69 2011). Notably, the innermost part of the IC (IMIC) was found to present distinct anisotropic behavior 
70 from the outer IC (OIC) (e.g., Ishii \& Dziewoński, 2002; Beghein \& Trampert, 2003; Cormier \&

71 Stroujkova, 2005; Stephenson et al., 2021). Studies in the last two decades reveal that the slowest direction

72 of compressional-wave propagation in the IMIC is tilted relative to the Earth's rotation axis, while in the

73 OIC, it is equatorial. But this contrast is not well constrained due to limited observations for IC central

74 part that require source-receiver pairs close to $180^{\circ}$.

76 More observational evidence is required to reconcile these properties and the different mechanisms behind

77 them. However, the existing observations are limited to IC compressional-wave anisotropy. Possible

78 anisotropy for IC shear waves ( $\mathrm{J}$ waves) that constrains the central part of the IC remains elusive. To our

79 best knowledge, there are minimal direct observations of J waves (Julian et al., 1972; Okal \& Cansi, 1998;

80 Deuss et al., 2000; Cao et al., 2005; Wookey \& Helffrich, 2008), likely because of the very weak amplitude

81 of J waves (Shearer et al., 2011).

83 Here we present a new observation of IC shear-wave anisotropy based on recent advances in global coda-

84 correlation wavefield (Phạm et al., 2018; Wang \& Tkalčić, 2020; Tkalčić et al., 2020). We observe stable

85 time and amplitude variations for the coda-correlation feature I2-J (Tkalčić \& Phạm, 2018) sensitive to J-

86 wave speed at the periods $15-50 \mathrm{~s}$ when its constituents sample the IC in different directions. The I2-J is

87 formed by pairs of seismic waves (Figures $1 \mathrm{~b}$ and $2 \mathrm{a}-\mathrm{c}$ ). We argue that the observed variations are due to

88 IC shear-wave anisotropy based on analyzing and eliminating multiple possible causes. We then evaluate

89 the J-wave anisotropy strength and examine various iron crystal models which can cause anisotropy. We

90 show that $\mathbf{J}$ waves traveling in directions oblique to the Earth's rotation axis travel faster than those

91 traveling in the equatorial plane. Although we cannot utilize $\mathrm{J}$ waves in planes parallel to the rotation axis 
92 and determine whether the hcp or bcc iron model dominates the IC from our observations alone, we show

93 that we can rule out one bcc model from many candidate models.

(a) Seismic wavefield observations

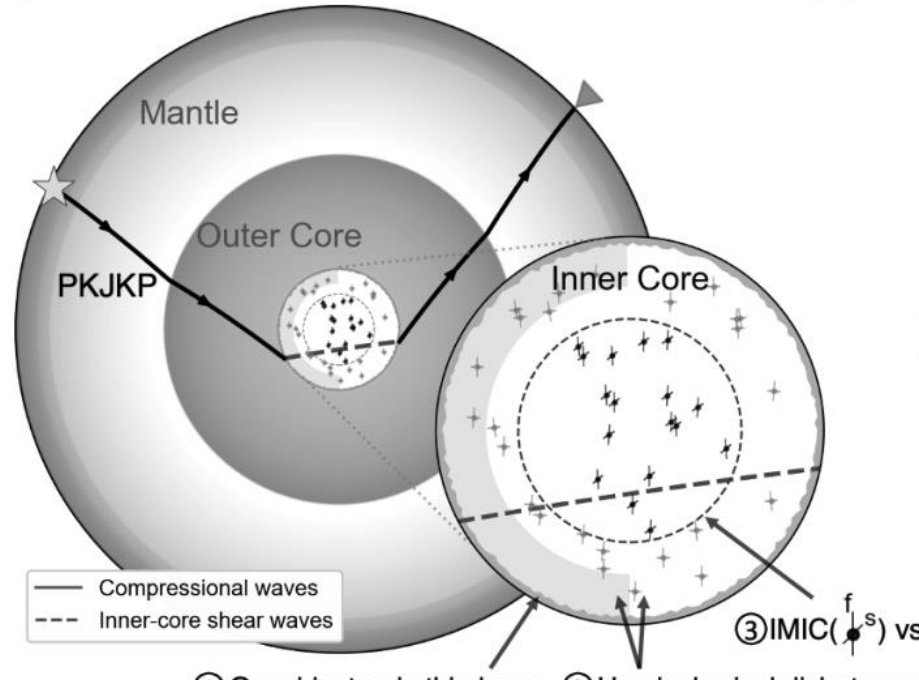

(1) Quasi-isotropic thin layer (2)Hemispherical dichotomy (b) Coda-correlation wavefield observations

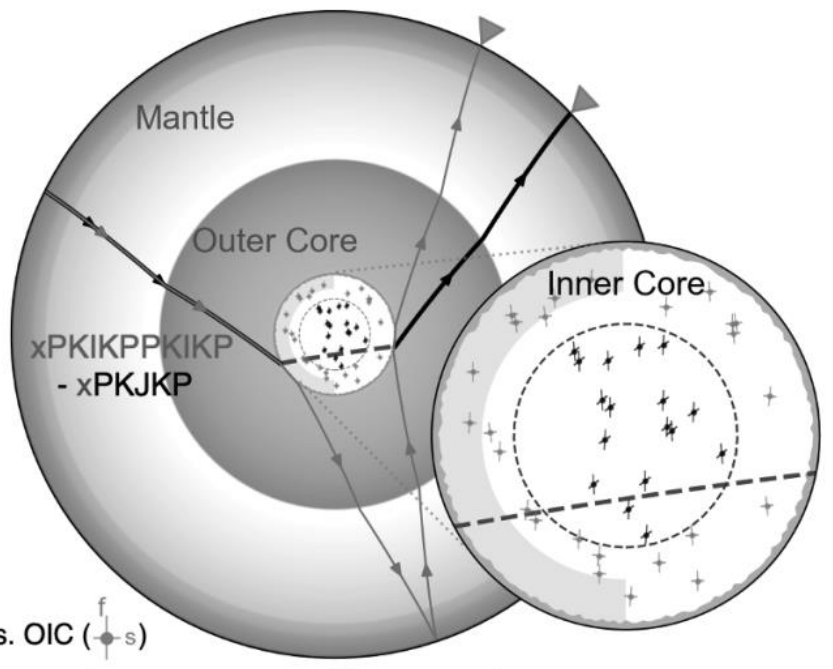

(4) LPO iron of hcp (纤) or bcc ( ) ?

Figure 1. Complex anisotropic IC sampled by seismic wavefield and coda-correlation wavefield. The observed complexities shown in the insets are explained in the main text. (a) Ray path for a PKJKP wave from an event (star) to a receiver (triangle). The dashed line indicates the IC shear wave (J wave). (b) A diagram for correlation feature I2-J formed due to the similarity between two seismic waves: xPKIKPPKIKP (xI2) and xPKJKP (xJ) recorded at two receivers (triangles), respectively. " $\mathrm{x}$ " in the nomenclature represents any common combination of ray legs for the two seismic waves and is not shown. The dashed line indicates the $\mathrm{J}$ wave for the pair xI2-xJ. Other pairs of seismic waves contributing to I2-J and the resultant diverse J-wave directions are explained in the main text and shown in Figure 2.

\section{The Observations and Confirmation of J-wave Anisotropy from Coda-correlation Wavefield}

We compute earthquake coda-correlation wavefields for I2-J features following Phạm et al. (2018) and Wang and Tkalčić (2020). The I2-J is formed due to the similarity of seismic waves in a plane proximal to the great-circle plane defined by a receiver pair (Tkalčić \& Phạm, 2018; Wang \& Tkalčić, 2020). Multiple seismic waves can contribute to forming PKIKPPKIKP-PKJKP (I2-J) in a great-circle plane, such as xPKIKPPKIKP-xPKJKP (xI2-xJ) and xPKIKPPKIKPPKIKP-xPKJKPPKIKP (xI3-xJI) (Figures 2a-c), in which "x" represents the common ray legs for a pair of seismic waves. Therefore, we cannot 
111 uniquely define the direction for an individual J-wave ray path for the feature I2-J. Accordingly, we bin 112 great-circle planes based on the angle $\varphi$ for which $90^{\circ}-\varphi$ is the angle between the plane's normal and 113 the Earth's rotation axis. Figures 2 d-f showcase quasi-equatorial $\left(\varphi \sim 90^{\circ}\right)$, oblique $\left(\varphi \sim 50^{\circ}\right)$, and quasi114 polar $\left(\varphi \sim 0^{\circ}\right)$ planes. In each of the planes, J-wave directions vary. In the quasi-equatorial plane, J-wave 115 directions are exclusively quasi-equatorial. J-wave directions can range from equatorial to oblique in the 116 oblique plane, and the more oblique the plane gets, the more versatile J-wave directions become. In the 117 quasi-polar plane, $\mathrm{J}$ waves can take arbitrary directions.

119 We then select the events with hypocenters proximal to the great-circle planes (Figures $2 \mathrm{~g}-\mathrm{i}$ ). We 120 empirically select events close to the great-circle path for a spherical distance smaller than $15^{\circ}$, and 121 exclude those farther than $15^{\circ}$ (Wang \& Tkalčić, 2020). After the selection, for specific $\varphi$, the I2-J is 122 predominantly sensitive to the Earth structure proximal to the great-circle plane (Wang \& Tkalčić, 2020). 123 We consider global events regardless of their epicentral distances. Any event close to the great-circle plane 124 can contribute to forming the correlation feature I2-J (Figures 2a-c). Subsequently, we compute coda125 correlation stacks for different $\varphi$ ranges, with a step of $10^{\circ}$ (Figure S4; see Methods section in the 126 supporting information), and we conclude that I2-J is not prominently visible in all ranges. We, therefore, 127 compute stacks for $20^{\circ}$-wide bins (Figures $\left.3 a-b\right)$.

129 As shown in Figure 3, I2-J exhibits a variation in time for different $\varphi$ ranges. We find that the I2-J timing 130 for the bin $\varphi=40^{\circ}-60^{\circ}$ (oblique planes) lags $\sim 5 \mathrm{~s}$ behind the bin $\varphi=60^{\circ}-80^{\circ}$ (quasi-equatorial planes) 131 (Figures 3a-d) via slant-stack analyses (see Methods section in the supporting information). This time 132 variation is robust given that the I2-J is a stacked cross-correlation feature based on ten-year recordings 133 for globally distributed events and receivers (Figure S1). We test the stability of time variations by 
134 analyses with denser bins (Figures 3g-i, S6, and S7) and bootstrap experiments (Figure S8 and Methods

135 section in the supporting information).

136

(a)

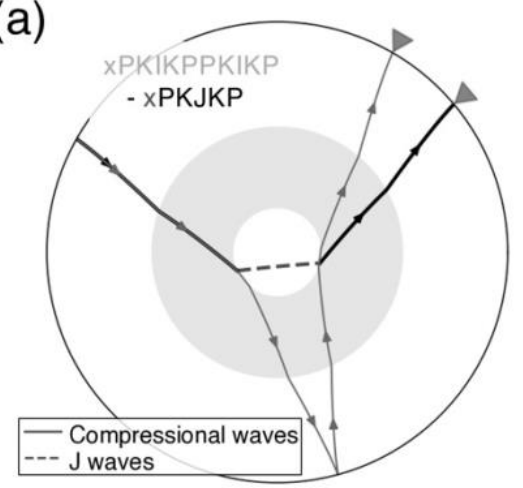

(d)

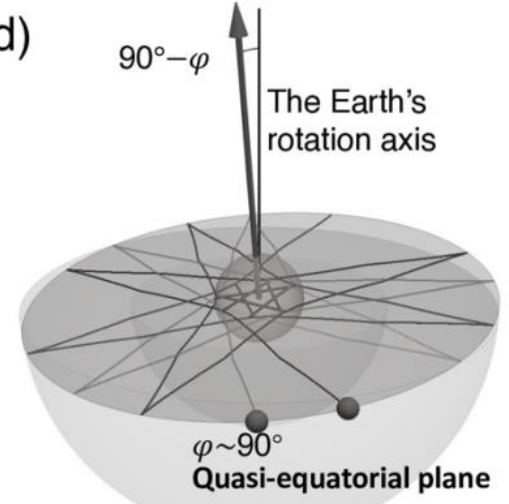

(g)

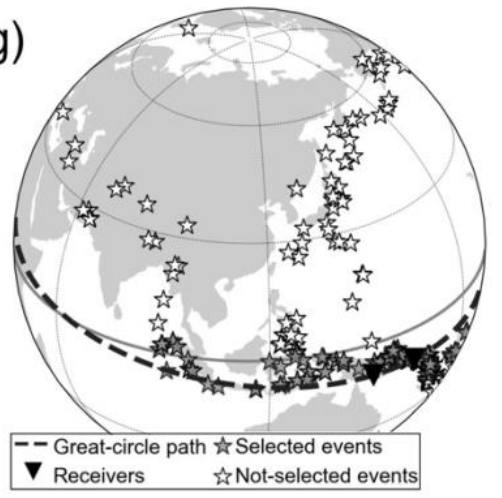

(b)

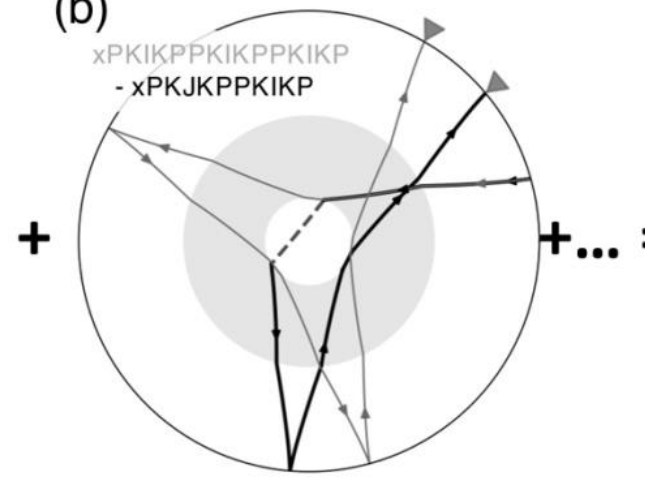

(e)

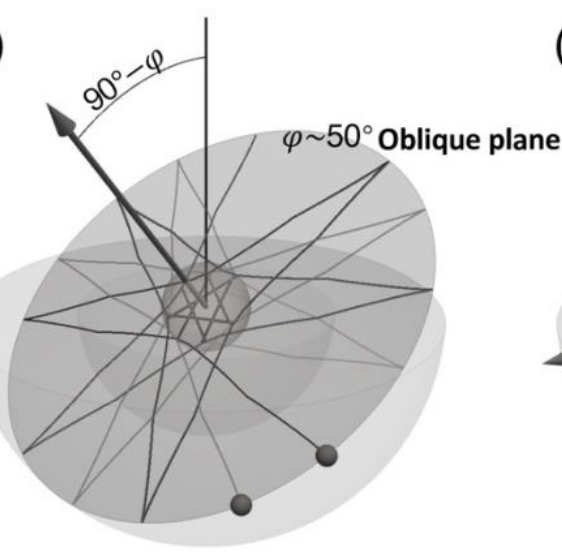

(h)

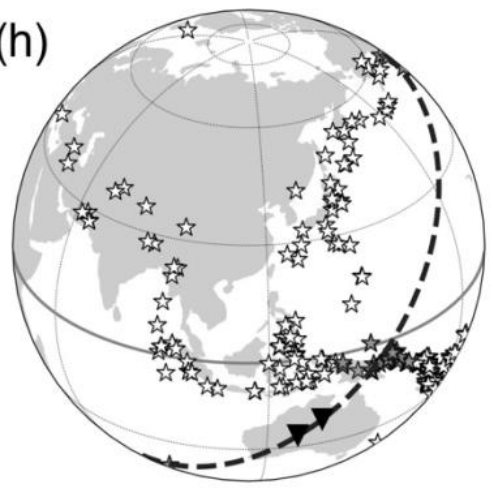

(c)

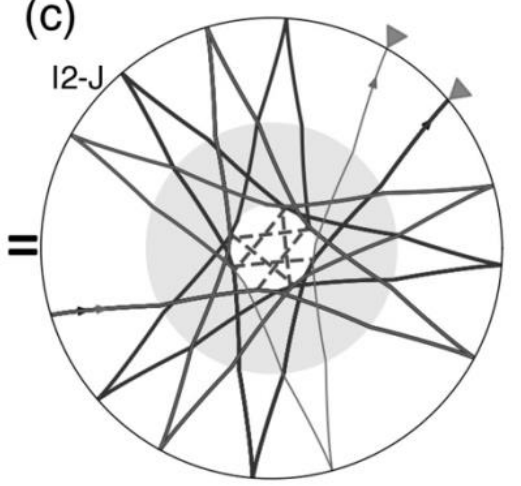

(f)
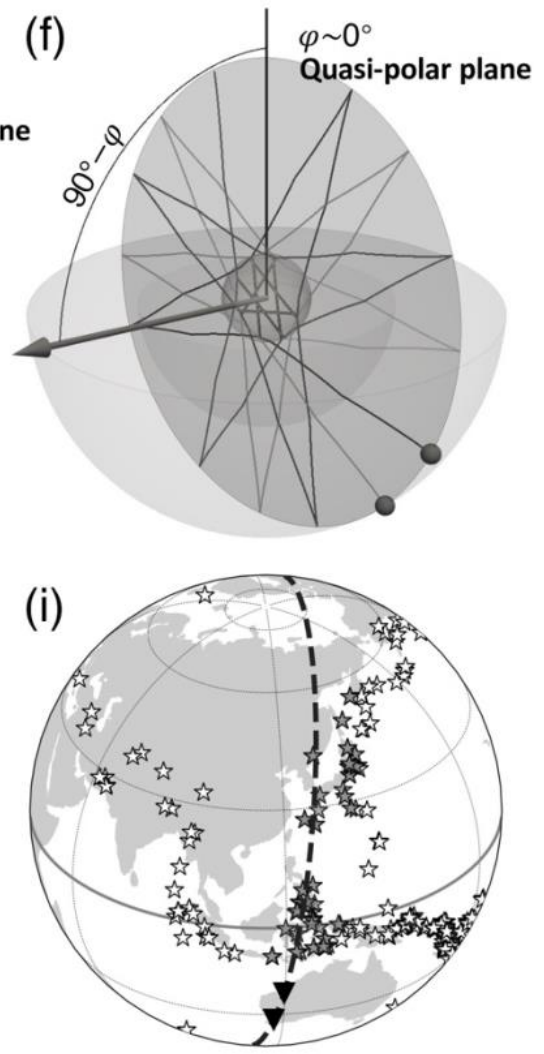

138 Figure 2. The ray-path geometry of the coda-correlation feature I2-J. (a) The I2-J can be formed by the 139 interference between two seismic waves: xPKIKPPKIKP (xI2) and xPKJKP (xJ), recorded at two 140 receivers (triangles), respectively. The " $\mathrm{x}$ " represents the common propagation legs for the two waves.

141 The dashed line represents the IC shear waves (J waves). (b) The I2-J formed by another two seismic 142 waves: xPKIKPPKIKPPKIKP (xI3) and xPKJKPPKIKP (xJ). (c) Combined (a), (b) plus other pairs not 
143 shown in a) and b) (xI4-xJI2, xI5-xJI3, xI6-xJI4, xI7-xJI5) that contribute to the correlation feature I2-J. 144 (d) I2-J ray paths inside a quasi-equatorial great-circle plane $\left(\varphi \sim 90^{\circ}\right)$. The plane passes through two 145 receivers (black balls). The angle between the plane's normal (black arrow) and the Earth's rotation axis 146 is defined as $90^{\circ}-\varphi$. All J-wave ray paths in the quasi-equatorial plane are in quasi-equatorial directions 147 (relative to the Earth's rotation axis). (e) Same as d) but for an oblique plane $\left(\varphi \sim 50^{\circ}\right)$. The J-wave ray 148 paths exhibit a range of equatorial and oblique directions relative to the Earth's rotation axis. (f) Same as $149 \mathrm{~d})$ but for a quasi-polar plane $\left(\varphi \sim 0^{\circ}\right)$. The J-wave ray paths are in arbitrary directions relative to the Earth's 150 rotation axis. (g) An example showing a selection of events (stars) for a quasi-equatorial great-circle plane 151 for two receivers (black triangles). We select events within the spherical distance of $15^{\circ}$ to the great-circle 152 path and exclude those farther than $15^{\circ}$. (h, i) Similar to $(\mathrm{g})$ but for oblique and quasi-polar planes, 153 respectively.

(a)

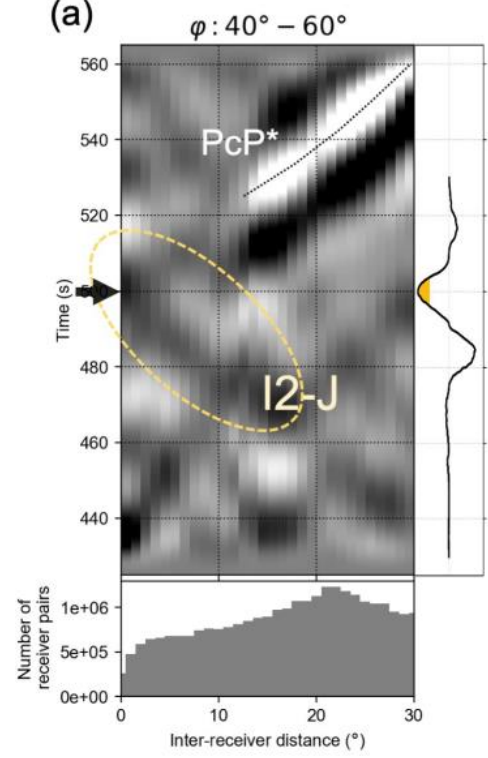

(b) $\quad \varphi: 60^{\circ}-80^{\circ}$

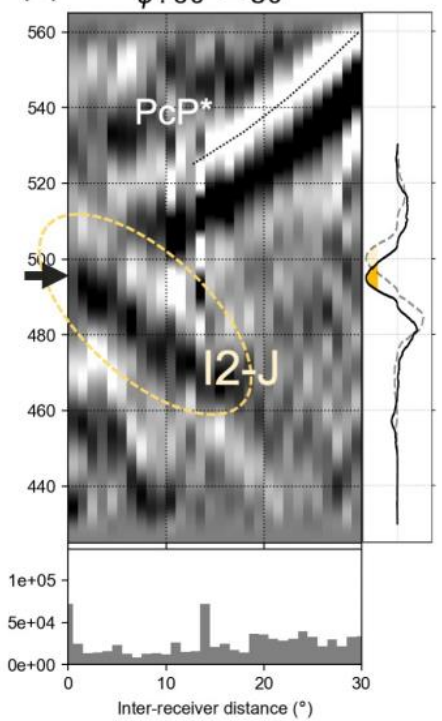

(g)

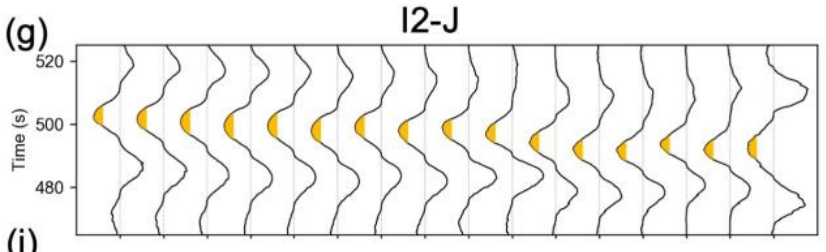

(i)

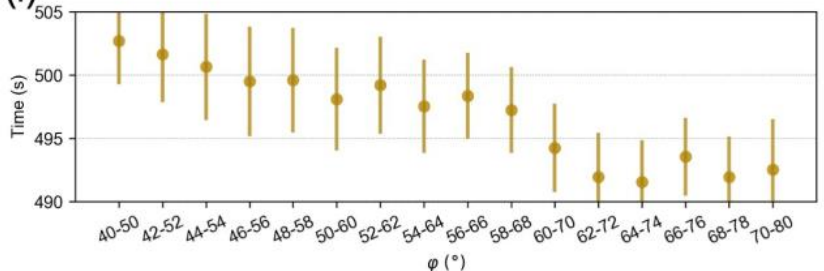

(c)
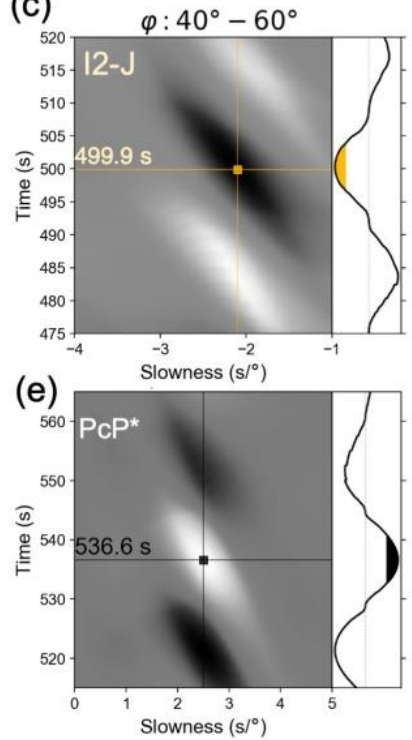

(d)

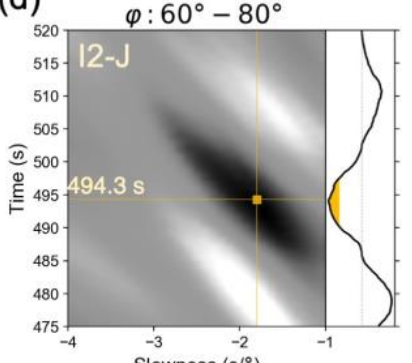

(f)

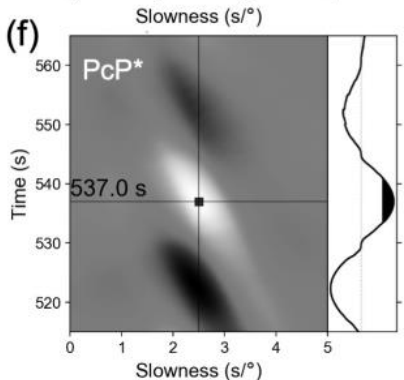

$\mathrm{PcP}^{*}$

(h)

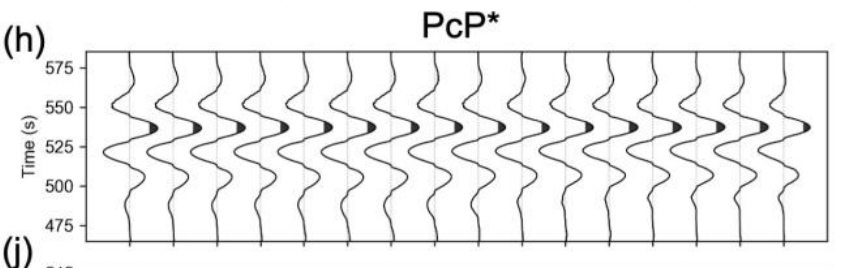

(j)

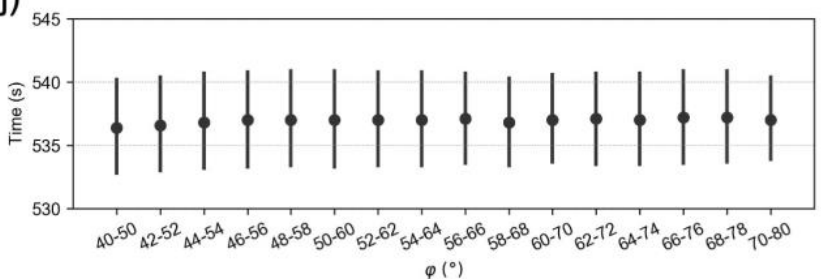


157 Figure 3. Observations of IC shear-wave anisotropy in coda-correlation wavefield. (a, b) Coda158 correlograms and frequency histograms of receiver pairs in different inter-receiver distance bins for two $159 \varphi$ ranges: (a) $40^{\circ}-60^{\circ}$, (b) $60^{\circ}-80^{\circ} . \varphi$ is defined in Figure 2; yellow ellipses and black arrows indicate the 160 coda-correlation feature I2-J sensitive to the IC shear-wave speed; dotted lines indicate the feature PcP*. 161 Positive correlation amplitudes are in white shades, and negative are in black shades; the intensity of the 162 black or white indicates the amplitude strength. The I2-J waveform stacks are shown alongside the 163 correlograms. (c, d) Slant stacks of I2-J for the two different $\varphi$ ranges, $40^{\circ}-60^{\circ}$, and $60^{\circ}-80^{\circ}$, respectively. 164 The yellow dots correspond to the lower branch of the I2-J cusp. Each slant stack is normalized with respect to the maximal amplitude. The I2-J waveform stacks are shown alongside the slant stacks. (e, f) Similar to (c, d) but for $\mathrm{PcP}^{*}$. $(\mathbf{g}, \mathbf{h}) \mathrm{I} 2-\mathrm{J}$ and $\mathrm{PcP}^{*}$ waveform stacks based on slant stacks for different $\varphi$ ranges. Colored areas correspond to stack amplitudes greater than $80 \%$ of the maximum. (i, j) Time measurements of the I2-J and PcP* waveform stacks. Dots correspond to the stacks' maximum amplitudes. Vertical bars correspond to the time range of the colored area in $(\mathrm{g}, \mathrm{h})$.

To explain the time variation, we consider multiple possibilities, similar in the scope to analyses of IC compressional-wave travel times (e.g., Poupinet et al., 1983; Morelli et al., 1986; Shearer et al., 1988; Creager, 1992). The possible explanations for our observations are 1) mantle structure, core-mantleboundary (CMB) topography, and ellipticity of the Earth, 2) outer-core (OC) heterogeneity, 3) IC 175 heterogeneity, 4) IC compressional-wave anisotropy, and 5) IC shear-wave anisotropy. In short, these 176 considerations show that the shear-wave anisotropy is the simplest and most likely cause for the observed 177 travel time variations.

179 Considering the first cause, the correlation stacks for each $\varphi$ range correspond to a laterally-averaged 180 mantle due to the mixture of raypaths at diverse directions (Figure 2). Hence, the signature from mantle 181 heterogeneity, CMB topography, and Earth's ellipticity is averaged out. This is evident via the correlation 182 feature $\mathrm{PcP}^{*}$, sensitive to the mantle structure, CMB topography, and Earth's ellipticity. Namely, for 183 different $\varphi, \mathrm{PcP}^{*}$ is nearly invariant compared to I2-J (Figures 3g-j), that the measured time variation for 
$184 \mathrm{PcP}^{*}$ is less than $0.5 \mathrm{~s}$ (Figures 3e-f). In conclusion, mantle structure, CMB topography, or Earth's 185 ellipticity cannot reconcile such a difference between I2-J and PcP*.

187 As far as the OC heterogeneity is considered, effects due to possible OC structure are mitigated due to the 188 diverse I2-J ray paths in the OC (Figure 2). Notably, the bulk of the OC is well-mixed and homogeneous 189 due to vigorous convection (Stevenson, 1987). Romanowicz et al. (2003) showed that OC tangent-cylinder 190 structure could explain PKP observations, although this was later disputed (e.g., Souriau et al., 2003; Ishii 191 and Dziewoński, 2005). Therefore, the possibility of a complex structure in the OC affecting I2-J is even 192 smaller than 1) to explain the I2-J variations.

194 IC heterogeneity requires significant structural anomalies in the bulk of the IC. If such anomaly 195 distribution indeed exists, there should also be evidence in compressional-wave travel time observations. 196 However, that has not been observed for the bulk of the IC (Shearer, 1994). The observed lateral variation 197 in travel times for the waves sampling the upper parts of the IC (Tanaka \& Hamaguchi, 1997; Niu \& Wen, 198 2001; Yee et al., 2014) is not sufficiently large to explain our observations. In conclusion, IC heterogeneity 199 would have to be more complex than the IC shear-wave anisotropy.

200 The IC has been hypothesized to be cylindrically anisotropic for compressional waves based on seismic 201 travel times (Morelli et al., 1986; Shearer et al., 1988; Creager, 1992) and normal modes (Woodhouse et 202 al., 1986; Tromp, 1993). PKIKP waves propagate faster along the rotation axis than along equatorial 203 directions. However, the IC compressional-wave anisotropy cannot explain the observed time difference 204 for I2-J. The IC compression waves (I-wave legs) contributing to I2-J do not penetrate deep into IC. The 205 I waves sample the topmost IC at a maximal depth less than $10 \mathrm{~km}$ below the IC boundary (ICB) (Figure $206 \mathrm{~S} 11$ ), and the total travel time for the I legs is less than $50 \mathrm{~s}$. Therefore, if the IC compressional-wave 
207 anisotropy were the cause for our observations, it would have to be at least $10 \%$ in the topmost $10 \mathrm{~km}$ of 208 the IC, which has not been observed. Quite to the contrary, a thin layer in the upper IC is revealed to be 209 quasi-isotropic (e.g., Shearer, 1994; Song \& Helmberger, 1995).

211 The IC can be anisotropy for shear waves due to lattice-preferred orientation (LPO) of iron crystal in either 212 hcp (Steinle-Neumann et al., 2001; Stixrude \& Cohen, 1995) or bcc (Belonoshko et al., 2008; Calvet \& 213 Margerin, 2008; Vočadlo et al., 2003) models in the IC hypothesized for explaining the compressional214 wave anisotropy. By invoking these models, theoretical computations predict travel-time difference up to 215 a few tens of seconds for J waves sampling the whole bulk of the IC in different directions (Song, 1997; 216 Stixrude \& Cohen, 1995; Vočadlo et al., 2009). That is sufficient to explain the observed $\sim 5 \mathrm{~s}$ time 217 difference via invoking a portion of LPO of iron inside the IC. The J-wave anisotropy might be different 218 in the OIC and the IMIC because the same is true for the P-wave anisotropy (e.g., Ishii \& Dziewoński, 219 2002; Beghein \& Trampert, 2003; Cormier \& Stroujkova, 2005; Stephenson et al., 2021). However, from 220 the observations of I2-J, we cannot distinguish between the OIC and the IMIC. As shown in Figure S11, 221 the $\mathrm{J}$ waves contributing to I2-J sample the bulk of the IC from the ICB to depth $\sim 815 \mathrm{~km}$ below the ICB, 222 and that is similar for all I2-J at different inter-receiver distances. Therefore, the observed $\sim 5 \mathrm{~s}$ time 223 difference for I2-J corresponds to an aggregated J-wave anisotropy for the whole bulk of the IC.

$225 \mathrm{~J}$-wave anisotropy is also supported by the varied strength of the I2-J cusp for different angle $\varphi$ ranges. 226 The I2-J cusp presents weaker amplitude for $\varphi$ in the range $40^{\circ}-60^{\circ}$ than in $60^{\circ}-80^{\circ}$ (Figures $3 \mathrm{a}-\mathrm{b}$ ), and 227 loses its visibility when $\varphi$ approaches $0^{\circ}$ although there are still a large number of cross-correlation pairs 228 (Figure $\mathrm{S} 4$ ). When $\varphi$ is close to $0^{\circ}$, the $\mathrm{J}$ waves contributing to I2-J sample the IC at varying angles relative 229 to the Earth's rotation axis (Figure 2f) and hence have notable different travel times due to anisotropy. 
230 Namely, the time difference due to shear-wave anisotropy can be up to tens of seconds, as predicted (Song,

231 1997; Stixrude \& Cohen, 1995; Vočadlo et al., 2009). The time difference between J waves can decrease

232 the amplitude of their stacks when forming I2-J (Wang \& Tkalčić, 2020). To test the effect of anisotropy

233 on the strength of I2-J, we perform a synthetic experiment (see Methods section in the supporting

234 information). As shown in Figure S10, J waves interfere destructively for polar and polar-oblique planes, 235 yielding a decrease in the amplitude of I2-J stacks. The observations confirm that.

237 In contrast, since all $\mathrm{J}$ waves have sufficiently similar travel times for the equatorial and quasi-equatorial 238 planes, they constructively interfere to form a clear I2-J feature. That is evident by the strong I2-J 239 amplitude for $\varphi$ in $60^{\circ}-80^{\circ}$ although the number of receiver pairs is less than one-tenth of those at other $240 \varphi$ ranges (Figures 3a-b and S4). We cannot rule out attenuation anisotropy in the IC (Mäkinen et al., 2014; 241 Souriau \& Romanowicz, 1996), which would also contribute to explaining the unclear I2-J feature. Strong 242 attenuation in polar directions would weaken a portion of $\mathrm{J}$ waves constituting I2-J for $\varphi$ close to $0^{\circ}$. But 243 for $\varphi$ close to $90^{\circ}$, the constituents of I2-J do not suffer from the strong attenuation effects, and their stacks 244 result in visible I2-J features.

2463 The Anisotropy Strength and Implications for the Stable Phase of Iron and Viscosity

247 Based on the above rationale, we evaluate the IC shear-wave cylindrical anisotropy strength by defining 248 the angle $\xi$ as the angle between the individual J-wave raypath and the Earth's rotation axis (Figure 4a; 249 Shearer et al., 1988; Tkalčić, 2015). For I2-J in a plane defined by $\varphi$, the angles $\xi$ of contributing J waves 250 are distributed in the interval from $\varphi$ to $90^{\circ}$ (Figure 2). The observed I2-J travel time corresponds to an 251 averaged $\mathrm{J}$ wave at those different $\xi$ angles. We evaluate that the $\mathrm{J}$ waves are faster for at least $\sim 5 \mathrm{~s}$ at the 252 oblique angles $\left(\xi=40^{\circ}-60^{\circ}\right)$ than at the equatorial angles $\left(\xi=60^{\circ}-80^{\circ}\right)$ (see Methods section in the 
253 supporting information). The $\sim 5 \mathrm{~s}$ time difference sets a minimum bound of shear-wave anisotropy 254 strength to $\sim 0.8 \%$. We do not have enough resolution for polar angles due to the unclear I2-J when $\varphi$ 255 approaches $0^{\circ}$ (Figure $\mathrm{S} 4$ ). The $\sim 0.8 \% \mathrm{~J}$-wave anisotropy is comparable to the $\sim 1 \%$ based on direct seismic 256 body wave observations (Wookey \& Helffrich, 2008).

258 Unlike some other coda-correlation features (e.g., I2* in Wang \& Tkalčić (2020)), I2-J cannot be separated 259 into unambiguous (timing-wise) constituents (Wang \& Tkalčić, 2020). Hence, we cannot determine the 260 travel times of individual $\mathrm{J}$ waves along different raypaths. This prevents us from observing J-wave 261 splitting into two polarized shear waves (quasi-SV and quasi-SH) propagating at different speeds. 262 Furthermore, the quasi-SH in the IC can be relatively weaker than the quasi-SV because of its ineffective 263 conversion from and to the $\mathrm{P}$ waves at the ICB. Nevertheless, the J-wave observations derived from coda264 correlation stacks correspond to an average of two split shear waves.

266 Various crystalographic models have been suggested to explain the cylindrical anisotropy of the IC based 267 on compressional-wave observations, as summarized in many studies (e.g., Mattesini et al., 2010; 268 Romanowicz et al., 2016; Vočadlo et al., 2009). Although we cannot obtain an evaluation for J-wave 269 anisotropy based on the full range of angles $\xi$, we can check each model's compatibility with our 270 observations. We consider the following models: hcp with its $c$ axis quasi-parallel to the Earth's rotation 271 axis (Figure 4a) and bcc with different crystal alignments (bcc001, bcc110, and bcc111, shown in Figures 272 4b-d). We calculate averaged J-wave velocities by assuming a single crystal for the whole IC (see Methods 273 section in the supporting information). As shown in Figure 4e, the bcc001 model predicts slower $\mathbf{J}$ waves 274 at oblique $\left(\xi=40^{\circ}-60^{\circ}\right)$ than equatorial directions $\left(\xi=60^{\circ}-90^{\circ}\right)$. That is the opposite of our observation. In 275 contrast, the hcp, bcc110, and bcc111 models predict faster $\mathrm{J}$ waves at oblique than equatorial directions, 
276 in agreement with our observation. Therefore, bcc001 can be excluded from candidate IC iron models.

277 However, we cannot distinguish between the hcp, bcc110, and bcc111 models because they give similar

278 travel time curves (Figure 4e). This is somewhat similar to the conclusion drawn based on direct body 279 wave observations at shorter periods (Wookey \& Helffrich, 2008), in which different models yield similar

280 J-wave anisotropy. Both this study and Wookey \& Helffrich (2008) rely on theoretical computations of 281 elasticity for iron crystals. Wookey \& Helffrich (2008) prefers an hcp model with its $c$ axis perpendicular 282 to the Earth's rotation axis considering the fastest P-waves at directions perpendicular to the $c$ axis 283 (Steinle-Neumann et al., 2001), however opposite anisotropic properties for the hcp iron were reported by 284 different studies, related to temperature and pressure uncertainty (e.g., Antonangeli et al., 2006; Stixrude 285 \& Cohen, 1995; Vočadlo et al., 2009).

287 Furthermore, the predictions for single crystals exhibit much stronger anisotropy than the observations. A 288 portion of the iron crystal's LPO, related to crystal defects or grain boundaries, could decrease the 289 anisotropy strength. We set a model for imperfect crystal alignment (bcc111-G in Figure 4e). We set a 290 Gaussian distribution of the cubic main diagonal orientations around the Earth's rotation axis, and the 291 Gaussian distribution has its half maximum at $30^{\circ}$. This imperfect crystal alignment reduces the anisotropy 292 strength from $25.1 \%$ (bcc111) to $10.8 \%$ (bcc111-G). The crystal imperfections are also suggested to be a 293 reason for the lower rigidity of the IC in observations than in experimental predictions (Belonoshko et al., 294 2007). 
(a)

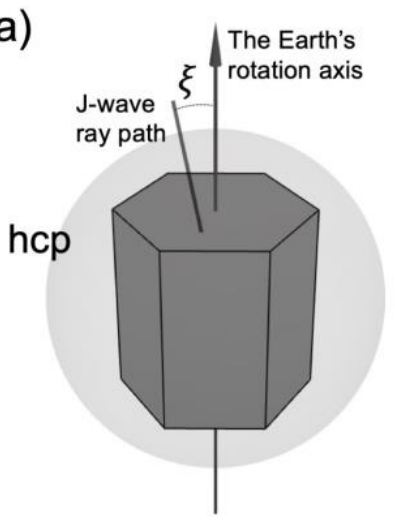

(b)

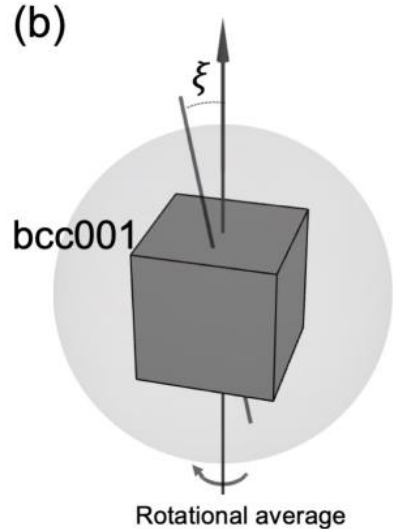

(c)

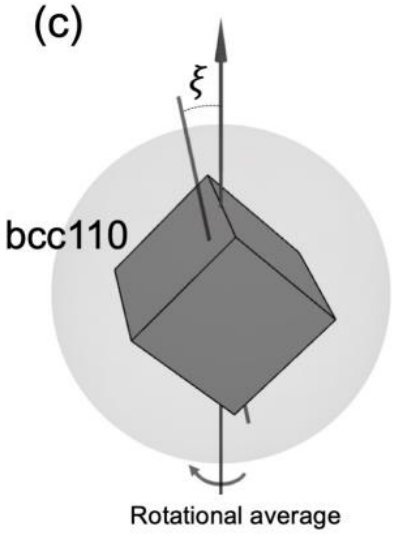

(d)

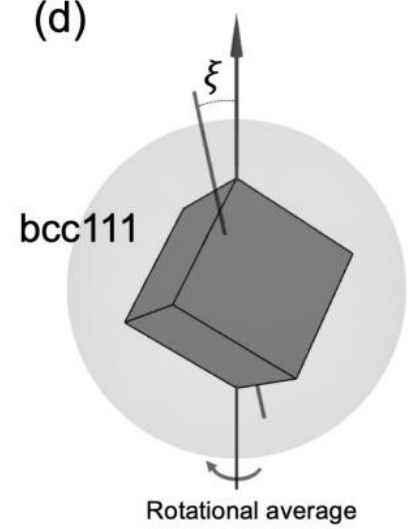

(e)

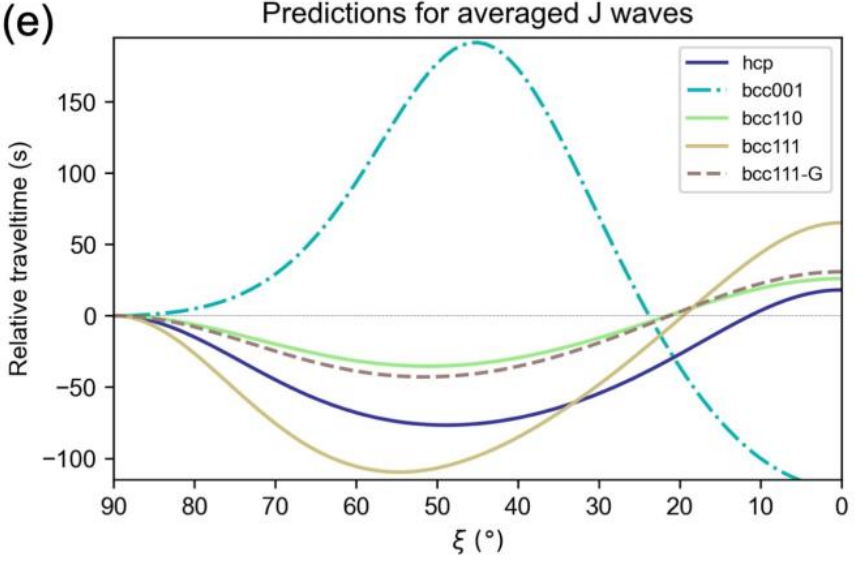

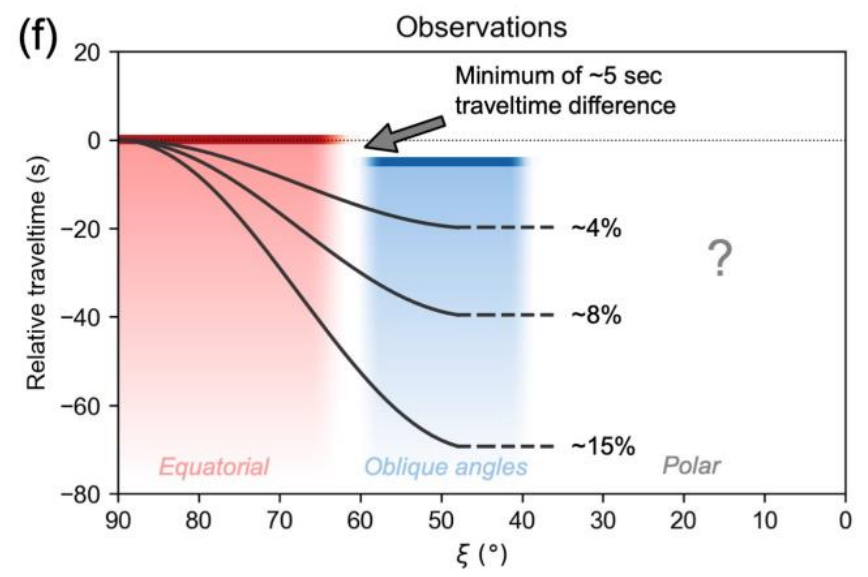

Figure 4. Hcp and bcc models for IC shear-wave anisotropy. (a-d) Diagrams of hcp and bcc models for iron crystal in the IC. $\xi$ is the angle between J-wave ray paths and the Earth's rotation axis. The hop model has its $c$ axis parallel to the Earth's rotation axis. The bcc001, bcc110, and bcc111 have the cube edge, face diagonal, and main diagonal parallel to the rotation axis, respectively. (e) J-wave travel time predictions as a function of the angle $\xi$ for the models shown in (a-d). bcc111-G corresponds to an imperfect crystal alignment for bcc111 (see main text). Elastic properties for different models are listed in Table S1. (f) Illustration of the observed relative travel times in this study. The curves indicate shear-wave anisotropy models of different strengths.

The analyses above are based on the J-wave speed variation between oblique and equatorial directions.

We lack constraints in polar directions due to unclear I2-J in polar planes ( $\varphi$ close to $0^{\circ}$ ). In polar planes, J waves contributing to I2-J sample the IC at varying angles $\left(\xi=0^{\circ}-90^{\circ}\right)$ and thus have highly variable 
309 (Wang \& Tkalčić, 2020). A more complex asymmetric IC model departing from the cylindrical anisotropy

310 (Romanowicz et al., 1996) can increase travel time variability and further decrease the amplitude of I2-J.

311 The anisotropy asymmetry may imply a slow translation in addition to the preferential equatorial growth

312 of the IC (Romanowicz et al., 1996; Frost et al., 2021). Currently, the coda-correlation observations cannot

313 provide constraints on some proposed complex models, but they do not rule out models of asymmetric

314 anisotropy and large-scale low-order convection in the IC ( Romanowicz et al., 1996; Frost et al., 2021).

315 A delicate grouping of I2-J with respect to longitude may shed light on the asymmetric anisotropy; 316 however, care should be taken due to the unequal distribution of great-circle planes as a function of 317 longitude (Figure S3).

319 The IC shear-wave anisotropy can bias estimation of the attenuation structure, which is an essential 320 parameter in understanding the viscosity and the related mineral physics and dynamics of the IC (Souriau 321 \& Romanowicz, 1996; Tkalčić \& Phạm, 2018). For example, the attenuation strength can be overestimated 322 from the coda-correlation wavefield observations if anisotropy is not considered, as noted by Tkalčić \& 323 Pham (2018). The amplitude of a correlation feature can decrease due to the stacking contributions 324 affected by anisotropic travel times (Wang \& Tkalčić, 2020). As shown in a synthetic experiment (Figure $325 \mathrm{~S} 10$ ), the anisotropy for a simple hcp model in the IC can result in amplitude reduction >80\% for I2-J 326 stacks from $\varphi=85^{\circ}$ to $\varphi=5^{\circ}$ without adding any attenuation effect.

\section{Conclusion}

329 We observe travel time and amplitude variations for coda-correlation feature I2-J, which confirms the 330 existence of shear-wave anisotropy in the bulk of the IC. The shear waves traverse the IC near its center 331 faster in directions oblique to the Earth's rotation axis than in the equatorial directions. The observed travel 
332 time difference is $\sim 5 \mathrm{~s}$, which translates to the apparent anisotropy strength of $\sim 0.8 \%$. However, given the

333 increasing versatility in J-wave raypath directions for the planes progressing from equatorial to polar

334 angles, we cannot utilize the quasi-polar planes, and $0.8 \%$ is the lower estimate of the anisotropy strength.

335 The anisotropy can be explained by invoking a portion of LPO of iron either in hcp or bcc structure.

336 Currently, our observations rule out the bcc001 structure, but other iron structures are not distinguishable.

337 A delicate grouping of coda-correlation stacks may help reveal a complex IC anisotropy. Further

338 proliferation of seismographs worldwide, including the ocean floor, will provide uniform coverage of 339 coda-correlation observations and resolve trade-offs between anisotropic and attenuative structures.

\section{Acknowledgments}

342 We are grateful to Dr. Phạm Thanh-Son and other Global Seismology group members at The Australian 343 National University for valuable discussions. We are also thankful to Prof. Maurizio Mattesini for advising 344 us on computations of anisotropic speeds for various iron crystal models. Seismic waveform data used in 345 this study are retrieved from IRIS DMC (https://ds.iris.edu/ds/nodes/dmc/data/) with ObspyDMT 346 (Hosseini \& Sigloch, 2017). The computations are performed at the National Computation Infrastructure 347 (NCI Australia) computer cluster as part of the ANUMAS grant \#em78. The Australian Government 348 provides the NCI infrastructure through its National Collaborative Research Infrastructure Strategy 349 (NCRIS). The authors declare no competing interests. 


\section{References}

Antonangeli, D., Merkel, S., \& Farber, D. L. (2006). Elastic anisotropy in hcp metals at high pressure and the sound wave anisotropy of the Earth's inner core. Geophysical Research Letters, 33(24). https://doi.org/10.1029/2006GL028237

Aubert, J., Amit, H., Hulot, G., \& Olson, P. (2008). Thermochemical flows couple the Earth's inner core growth to mantle heterogeneity. Nature, 454(7205), 758-761. https://doi.org/10.1038/nature07109

Auld, B. A. (1990). Acoustic fields and waves in solids. Vol. 1 Vol. 1. Malabar: Krieger Publishing Company.

Beghein, C., \& Trampert, J. (2003). Robust Normal Mode Constraints on Inner-Core Anisotropy from Model Space Search. Science, 299(5606), 552-555. https://doi.org/10.1126/science.1078159

Belonoshko, A. B., Skorodumova, N. V., Davis, S., Osiptsov, A. N., Rosengren, A., \& Johansson, B. (2007). Origin of the Low Rigidity of the Earth's Inner Core. Science, 316(5831), 1603-1605. https://doi.org/10.1126/science.1141374

Belonoshko, A. B., Skorodumova, N. V., Rosengren, A., \& Johansson, B. (2008). Elastic Anisotropy of Earth's Inner Core. Science, 319(5864), 797-800. https://doi.org/10.1126/science.1150302

Bergman, M. I. (1997). Measurements of electric anisotropy due to solidification texturing and the implications for the Earth's inner core. Nature, 389(6646), 60-63. https://doi.org/10.1038/37962

Biggin, A. J., Piispa, E. J., Pesonen, L. J., Holme, R., Paterson, G. A., Veikkolainen, T., \& Tauxe, L. (2015). Palaeomagnetic field intensity variations suggest Mesoproterozoic inner-core nucleation. Nature, 526(7572), 245-248. https://doi.org/10.1038/nature15523

Braginsky, S. I. (1963). Structure of the F layer and reasons for convection in the Earth's core. Soviet Phys. Dokl., 149, 8-10. 
381 Buffett, B. A., \& Wenk, H.-R. (2001). Texturing of the Earth's inner core by Maxwell stresses. Nature, 413(6851), 60-63. https://doi.org/10.1038/35092543

383 Buffett, Bruce A., Huppert, H. E., Lister, J. R., \& Woods, A. W. (1996). On the thermal evolution of the 384 Earth's core. Journal of Geophysical Research: Solid Earth, 101(B4), 7989-8006.

385 https://doi.org/10.1029/95JB03539

386 Cao, A. (2005). An Observation of PKJKP: Inferences on Inner Core Shear Properties. Science, 308(5727), 1453-1455. https://doi.org/10.1126/science.1109134

Calvet, M., \& Margerin, L. (2008). Constraints on grain size and stable iron phases in the uppermost inner core from multiple scattering modeling of seismic velocity and attenuation. Earth and Planetary Science Letters, 267(1), 200-212. https://doi.org/10.1016/j.epsl.2007.11.048

Chapman, C. H. (1981). Generalized Radon transforms and slant stacks. Geophysical Journal International, 66(2), 445-453. https://doi.org/10.1111/j.1365-246X.1981.tb05966.x

Cormier, V. F., \& Stroujkova, A. (2005). Waveform search for the innermost inner core. Earth and Planetary Science Letters, 236(1), 96-105. https://doi.org/10.1016/j.eps1.2005.05.016

Creager, K. C. (1992). Anisotropy of the inner core from differential travel times of the phases PKP and PKIKP. Nature, 356(6367), 309-314. https://doi.org/10.1038/356309a0

Deuss, A., Woodhouse, J. H., Paulssen, H., \& Trampert, J. (2000). The observation of inner core shear waves. Geophysical Journal International, 142(1), 67-73. https://doi.org/10.1046/j.1365246x.2000.00147.x constrained by seismic anisotropy. Nature Geoscience, 1-5. https://doi.org/10.1038/s41561-02100761-w 
403 Gubbins, D., Sreenivasan, B., Mound, J., \& Rost, S. (2011). Melting of the Earth's inner core. Nature, 404 473(7347), 361-363. https://doi.org/10.1038/nature10068

405 Hosseini, K., \& Sigloch, K. (2017). ObspyDMT: a Python toolbox for retrieving and processing large 406 seismological data sets. Solid Earth, 8(5), 1047-1070. https://doi.org/10.5194/se-8-1047-2017 407 Ishii, M., \& Dziewoński, A. M. (2002). The innermost inner core of the earth: Evidence for a change in 408 anisotropic behavior at the radius of about $300 \mathrm{~km}$. Proceedings of the National Academy of 409 Sciences, 99(22), 14026-14030. https://doi.org/10.1073/pnas.172508499

Ishii, M., \& Dziewoński, A. M. (2005). Constraints on the outer-core tangent cylinder using normalmode splitting measurements. Geophysical Journal International, 162(3), 787-792. https://doi.org/10.1111/j.1365-246X.2005.02587.x

Jeanloz, R., \& Wenk, H.-R. (1988). Convection and anisotropy of the inner core. Geophysical Research Letters, 15(1), 72-75. https://doi.org/10.1029/GL015i001p00072

Julian, B. R., Davies, D., \& Sheppard, R. M. (1972). PKJKP. Nature, 235(5337), 317-318. https://doi.org/10.1038/235317a0

421 Kennett, B. L. N., Engdahl, E. R., \& Buland, R. (1995). Constraints on seismic velocities in the Earth from traveltimes. Geophysical Journal International, 122(1), 108-124. https://doi.org/10.1111/j.1365-246X.1995.tb03540.x

424 Lehmann, I. (1936). P'. Publications Du Bureau CentralSeismologique International, Série A, Travaux 
426 Lin, J.-F., Mao, Z., Yavaş, H., Zhao, J., \& Dubrovinsky, L. (2010). Shear wave anisotropy of textured 427 hcp-Fe in the Earth's inner core. Earth and Planetary Science Letters, 298(3), 361-366. $428 \quad$ https://doi.org/10.1016/j.eps1.2010.08.006

429 Mäkinen, A. M., Deuss, A., \& Redfern, S. A. T. (2014). Anisotropy of Earth's inner core intrinsic $430 \quad$ attenuation from seismic normal mode models. Earth and Planetary Science Letters, 404, 354431 364. https://doi.org/10.1016/j.epsl.2014.08.009

432 Mao, W. L., Struzhkin, V. V., Baron, A. Q. R., Tsutsui, S., Tommaseo, C. E., Wenk, H.-R., et al. (2008). 433 Experimental determination of the elasticity of iron at high pressure. Journal of Geophysical 434 Research: Solid Earth, 113(B9). https://doi.org/10.1029/2007JB005229

435 Mattesini, M., Belonoshko, A. B., Buforn, E., Ramírez, M., Simak, S. I., Udías, A., et al. (2010). 436 Hemispherical anisotropic patterns of the Earth's inner core. Proceedings of the National 437 Academy of Sciences, 107(21), 9507-9512. https://doi.org/10.1073/pnas.1004856107

438 Morelli, A., Dziewonski, A. M., \& Woodhouse, J. H. (1986). Anisotropy of the inner core inferred from PKIKP travel times. Geophysical Research Letters, 13(13), 1545-1548.

441 Niu, F., \& Wen, L. (2001). Hemispherical variations in seismic velocity at the top of the Earth's inner core. Nature, 410(6832), 1081-1084. https://doi.org/10.1038/35074073

443 Okal, E. A., \& Cansi, Y. (1998). Detection of PKJKP at intermediate periods by progressive multi-

Phạm, T.-S., Tkalčić, H., Sambridge, M., \& Kennett, B. L. N. (2018). Earth’s Correlation Wavefield: Late Coda Correlation. Geophysical Research Letters, 45(7), 3035-3042. https://doi.org/10.1002/2018GL077244 
449 Poupinet, G., Pillet, R., \& Souriau, A. (1983). Possible heterogeneity of the Earth's core deduced from 450 PKIKP travel times. Nature, 305(5931), 204-206. https://doi.org/10.1038/305204a0

451 Romanowicz, B., Li, X.-D., \& Durek, J. (1996). Anisotropy in the Inner Core: Could It Be Due To Low452 Order Convection? Science, 274(5289), 963-966. https://doi.org/10.1126/science.274.5289.963

453 Romanowicz, B., Tkalčić, H., \& Bréger, L. (2003). On the origin of complexity in PKP travel time data. 454 In V. Dehant, K. C. Creager, S. Karato, \& S. Zatman (Eds.), Geodynamics Series (Vol. 31, pp. 31-44). Washington, D. C.: American Geophysical Union. https://doi.org/10.1029/GD031p0031

Romanowicz, B., Cao, A., Godwal, B., Wenk, R., Ventosa, S., \& Jeanloz, R. (2016). Seismic anisotropy in the Earth's innermost inner core: Testing structural models against mineral physics predictions. Geophysical Research Letters, 43(1), 93-100. https://doi.org/10.1002/2015GL066734

Shearer, P. M. (1994). Constraints on inner core anisotropy from PKP(DF) travel times. Journal of Geophysical Research: Solid Earth, 99(B10), 19647-19659. https://doi.org/10.1029/94JB01470

Shearer, P. M., Toy, K. M., \& Orcutt, J. A. (1988). Axi-symmetric Earth models and inner-core anisotropy. Nature, 333(6170), 228-232. https://doi.org/10.1038/333228a0

Shearer, P. M., Rychert, C. A., \& Liu, Q. (2011). On the visibility of the inner-core shear wave phase PKJKP at long periods. Geophysical Journal International, 185(3), 1379-1383. https://doi.org/10.1111/j.1365-246X.2011.05011.x

Song, X. (1997). Anisotropy of the Earth's inner core. Reviews of Geophysics, 35(3), 297-313.

469 Song, X., \& Helmberger, D. V. (1995). Depth dependence of anisotropy of Earth's inner core. Journal 470 of Geophysical Research: Solid Earth, 100(B6), 9805-9816. https://doi.org/10.1029/95JB00244 
471 Souriau, A., \& Romanowicz, B. (1996). Anisotropy in inner core attenuation: A new type of data to 472 constrain the nature of the solid core. Geophysical Research Letters, 23(1), 1-4.

473 https://doi.org/10.1029/95GL03583

474 Souriau, A., Teste, A., \& Chevrot, S. (2003). Is there any structure inside the liquid outer core?

475 Geophysical Research Letters, 30(11). https://doi.org/10.1029/2003GL017008

476 Steinle-Neumann, G., Stixrude, L., Cohen, R. E., \& Gülseren, O. (2001). Elasticity of iron at the

477 temperature of the Earth's inner core. Nature, 413(6851), 57-60.

$478 \quad$ https://doi.org/10.1038/35092536

479 Stephenson, J., Tkalčić, H., \& Sambridge, M. (2021). Evidence for the Innermost Inner Core: Robust

480 Parameter Search for Radially Varying Anisotropy Using the Neighborhood Algorithm. Journal $481 \quad$ of Geophysical Research: Solid Earth, 126(1), e2020JB020545.

482 https://doi.org/10.1029/2020JB020545

483 Stevenson, D. J. (1987). Limits on lateral density and velocity variations in the Earth's outer core.

484 Geophysical Journal International, 88(1), 311-319. https://doi.org/10.1111/j.1365-

485 246X.1987.tb01383.x

486 Stixrude, L., \& Cohen, R. E. (1995). High-Pressure Elasticity of Iron and Anisotropy of Earth’s Inner

487 Core. Science, 267(5206), 1972-1975. https://doi.org/10.1126/science.267.5206.1972

488 Tanaka, S., \& Hamaguchi, H. (1997). Degree one heterogeneity and hemispherical variation of 489 anisotropy in the inner core from $\mathrm{PKP}(\mathrm{BC})-\mathrm{PKP}(\mathrm{DF})$ times. Journal of Geophysical Research: $490 \quad$ Solid Earth, 102(B2), 2925-2938. https://doi.org/10.1029/96JB03187

491 Tkalčić, H. (2015). Complex inner core of the Earth: The last frontier of global seismology. Reviews of 492 Geophysics, 53(1), 59-94. https://doi.org/10.1002/2014RG000469 
493 Tkalčić, H. (2017). The earth's inner core: revealed by observational seismology. Cambridge ; New $494 \quad$ York, NY: Cambridge University Press.

495 Tkalčić, H., \& Phạm, T.-S. (2018). Shear properties of Earth’s inner core constrained by a detection of J 496 waves in global correlation wavefield. Science, 362(6412), 329-332.

497 https://doi.org/10.1126/science.aau7649

498 Tkalčić, H., Phạm, T.-S., \& Wang, S. (2020). The Earth’s coda correlation wavefield: Rise of the new $499 \quad$ paradigm and recent advances. Earth-Science Reviews, 208, 103285.

$500 \quad$ https://doi.org/10.1016/j.earscirev.2020.103285

501 Tromp, J. (1993). Support for anisotropy of the Earth's inner core from free oscillations. Nature, 366(6456), 678-681. https://doi.org/10.1038/366678a0

503 Vočadlo, L., Alfè, D., Gillan, M. J., Wood, I. G., Brodholt, J. P., \& Price, G. D. (2003). Possible thermal 504 and chemical stabilization of body-centred-cubic iron in the Earth's core. Nature, 424(6948), 505 536-539. https://doi.org/10.1038/nature01829

506 Vočadlo, L., Dobson, D. P., \& Wood, I. G. (2009). Ab initio calculations of the elasticity of hcp-Fe as a 507 function of temperature at inner-core pressure. Earth and Planetary Science Letters, 288(3), 508 534-538. https://doi.org/10.1016/j.eps1.2009.10.015

509 Wang, S., \& Tkalčić, H. (2020). Seismic event coda-correlation's formation: implications for global 510 seismology. Geophysical Journal International, 222(2), 1283-1294.

511 https://doi.org/10.1093/gji/ggaa259

512 Waszek, L., \& Deuss, A. (2011). Distinct layering in the hemispherical seismic velocity structure of 513 Earth's upper inner core. Journal of Geophysical Research: Solid Earth, 116(B12).

$514 \quad$ https://doi.org/10.1029/2011JB008650 
515 Wenk, H.-R., Matthies, S., Hemley, R. J., Mao, H.-K., \& Shu, J. (2000). The plastic deformation of iron 516 at pressures of the Earth's inner core. Nature, 405(6790), 1044-1047.

517 https://doi.org/10.1038/35016558

518 Woodhouse, J. H., Giardini, D., \& Li, X.-D. (1986). Evidence for inner core anisotropy from free $519 \quad$ oscillations. Geophysical Research Letters, 13(13), 1549-1552.

520 https://doi.org/10.1029/GL013i013p01549

521 Wookey, J., \& Helffrich, G. (2008). Inner-core shear-wave anisotropy and texture from an observation 522 of PKJKP waves. Nature, 454(7206), 873-876. https://doi.org/10.1038/nature07131

523 Yee, T.-G., Rhie, J., \& Tkalčić, H. (2014). Regionally heterogeneous uppermost inner core observed 524 with Hi-net array. Journal of Geophysical Research: Solid Earth, 119(10), 7823-7845. 525 https://doi.org/10.1002/2014JB011341

526 Yoshida, S., Sumita, I., \& Kumazawa, M. (1996). Growth model of the inner core coupled with the outer 527 core dynamics and the resulting elastic anisotropy. Journal of Geophysical Research: Solid 528 Earth, 101(B12), 28085-28103. https://doi.org/10.1029/96JB02700 


\section{Contents of this file}

Methods

Figures S1 to S11

Tables S1 and S2

\section{$548 \quad$ Methods}

549 Computation of earthquake coda-correlation wavefield

550 We compute earthquake coda-correlation wavefield following Phạm et al. (2018) and Wang and Tkalčić 551 (2020). First, for global events and receivers (Figure S1), we select late-coda recordings in 3-9 h after 552 events' origin time. We perform temporal normalization to suppress surface waves and spectral whitening 553 to balance energy across the entire frequency band (Phạm et al., 2018). Then, we compute cross554 correlation functions for recordings at each pair of receivers. We select the events close to the great-circle 
555 plane passing through the two receivers due to their dominant and constructive contribution to coda556 correlation's formation (Wang \& Tkalčić, 2020). We empirically discard the events being spherically 557 farther than $15^{\circ}$ from the great-circle plane (Figures $2 \mathrm{c}, \mathrm{e}, \mathrm{g}$ ). For two receivers at the same location, the 558 great-circle plane passes through the receivers and an event. Finally, we bin and stack the correlation 559 functions with respect to the inter-receiver distance and filter the stacked correlograms with a 15-50 s $560 \quad(0.02-0.067 \mathrm{~Hz})$, second-order, zero-phase bandpass filter.

\section{Grouping coda-correlation functions for different I2-J stacks}

564 We group correlation functions based on the geometry of events and receiver pairs. As shown in Figures 5652 and S2, we can define a great-circle plane passing through the two receivers for each receiver pair. For two receivers at the same location, the great-circle plane passes through the receivers and an event. For each great-circle plane, we can define an angle $\varphi$ for which $90^{\circ}-\varphi$ is the angle between the plane's normal and the Earth's rotation axis. We select receiver pairs having $\varphi$ in the same bin (Figure S3), and then we use those receiver pairs to compute I2-J stacks. For varied $\varphi$, we get different I2-J correlograms (Figures 3 and S4), and they sample the IC differently (Figure 2).

Figure S5 shows the histograms for the number of receiver pairs relative to angle $\varphi$ and inter-receiver distance. There is a cliff-like change for $\varphi$ crossing $\sim 56^{\circ}$. That is because there is a limited number of stations close to the equator. Most stations, especially the USArray stations, the stations in East Asia and Europe, are at medium and high latitudes (Figure S1). The great-circle plane defined by any two receivers cannot be close to the equator plane $\left(\varphi=90^{\circ}\right)$. Specifically, the southernmost stations of the USArray are at latitudes $\sim 30^{\circ} \mathrm{N}$ (Figure S1), and hence the largest $\varphi$ for great-circle-planes defined by any two USArray stations is $\sim 60^{\circ}$. Given the dominance of USArray stations, there is a sharp reduction near $56^{\circ}$ in Figure S5. The column spike for $\varphi \sim 69^{\circ}$ and inter-receiver distance $\sim 15^{\circ}$ corresponds to the cross-correlations between two networks. The two networks are at $\sim 20^{\circ} \mathrm{N}$ and are $\sim 15^{\circ}$ away from each other. Several local-scale networks match such criteria, such as the Arabian Peninsula and East Africa networks or Central America and the Caribbean Sea networks. 


\section{Slant-stack analysis}

We perform slant stacking to transform the coda-correlation wavefield in distance-time $(x-t)$ domain into slowness-delay time ( $\tau-p)$ domain (Chapman, 1981):

$$
s(\tau, p)=\sum_{i} c\left(\tau+p\left(x_{i}-x_{0}\right), x_{i}\right)
$$

in which $c(t, x)$ represents coda-correlation correlograms, and $s(\tau, p)$ the slant stacks. To form a slant stack, each waveform $c\left(t, x_{i}\right)$ at a distance $x_{i}$ is shifted in time by $p\left(x_{i}-x_{0}\right)$, and then all shifted waveforms are stacked together. The time shift depends on the reference distance $x_{0}$. For I2-J slant stacks, we choose $x_{0}=0^{\circ}$. We search for the maximal amplitude point $\left(\tau_{0}, p_{0}\right)$ that corresponds to the optimal stack. The $\tau_{0}$ and $p_{0}$ correspond to the time and slowness of I2-J at $0^{\circ}$, respectively. We choose $x_{0}=20^{\circ}$ for $\mathrm{PcP} *$ slant stacks and the resultant $\tau_{0}$ and $p_{0}$ correspond to the time and slowness of $\mathrm{PcP} *$ at $20^{\circ}$. Also, from two-dimensional slant stacks, we can extract the waveform stack $s\left(\tau, p_{0}\right)$ that are time series with the obtained $p_{0}$. The waveform stacks for I2-J and PcP* are shown in Figures 3, S6-S8.

The correlation feature I2-J has two branches in correlograms (Figure S4). We only use the lower branch of the I2-J cusp for slant-stacking analysis. That brings better accuracy and reliability for several reasons. As shown in Figure 3, the lower branch is visible in a larger distance range than the upper branch. Second, the lower branch is less contaminated by the strong feature $\mathrm{PcP} *$ than the upper branch. Third, the I2-J lower branch has a negative moveout while the $\mathrm{PcP}^{*}$ presents a positive one, making them distinctive in the $\tau-p$ domain.

\section{Bootstrap experiments}

Due to the complex composition of $\mathrm{J}$ waves in forming I2-J stacks, we perform bootstrap experiments to test the stability of time measurements from the coda-correlation wavefield. We re-compute correlograms 200 times with random samples of receiver pairs, and then for each correlogram, we compute slant stacks and waveform stacks for I2-J and PcP*. As shown in Figure S8, the I2-J time variation between $\varphi=40^{\circ}$ $60^{\circ}$ and $\varphi=60^{\circ}-80^{\circ}$ is stable. Similarly, the $\mathrm{PcP}^{*}$ time is stable, and it is nearly invariant between $\varphi=40^{\circ}$ $60^{\circ}$ and $\varphi=60^{\circ}-80^{\circ}$. 


\section{Evaluation of IC shear-wave speed at different directions}

617 We compute coda-correlation stacks for different $\varphi$ ranges (Figure 2). We note that for $\varphi$ angles varying 618 from the equatorial to polar, I2-J corresponds to J waves sampling the IC in fundamentally different ways 619 (Figures 2d,e,f). Namely, in a plane defined by $\varphi$, J waves propagating at different angles $\xi$ in the interval 620 from $\varphi$ to $90^{\circ}$ (Figures 2 and S2) contribute to the I2-J stack. The observed I2-J travel time corresponds 621 to an averaged $\mathrm{J}$ wave speed at different $\xi$ angles.

622

623 Specifically, for the bin $\varphi=40^{\circ}-60^{\circ}$, the I2-J is a result of $\mathrm{J}$ waves for $\xi=40^{\circ}-90^{\circ}$, and for the bin $\varphi=60^{\circ}-$ $62480^{\circ}$, the I2-J is a result of $\xi=60^{\circ}-90^{\circ}$. Accordingly, the $\sim 5 \mathrm{~s}$ time difference between $\varphi=40^{\circ}-60^{\circ}$ and $625 \varphi=60^{\circ}-80^{\circ}$ (Figure 3) represents J-wave travel-time difference between $\xi=40^{\circ}-90^{\circ}$ and $\xi=60^{\circ}-90^{\circ}$. For 626 that, $\mathrm{J}$ waves must be faster for at least $\sim 5 \mathrm{~s}$ at $\xi=40^{\circ}-60^{\circ}$ (oblique angles relative to the Earth's rotation 627 axis) than at $\xi=60^{\circ}-90^{\circ}$ (equatorial angles).

\section{Calculation of IC shear-wave speed}

631 We calculate the shear wave speed for an anisotropic IC with low-order harmonics approximations 632 following Song (1997):

$$
\rho V_{S 1}^{2}=C_{44}\left(\sin ^{4} \xi+\cos ^{4} \xi\right)+\left(C_{11}+C_{33}-2 C_{44}-2 C_{13}\right) \sin ^{2} \xi \cos ^{2} \xi,
$$

$$
\rho V_{S 2}^{2}=\frac{\left(C_{11}-C_{12}\right)}{2} \sin ^{2} \xi+C_{44} \cos ^{2} \xi,
$$

635 in which $V_{S 1}$ and $V_{S 2}$ are IC shear waves with polarization parallel to meridians and the equator, 636 respectively, $\rho$ the density, $\xi$ the angle between J-wave ray paths and the Earth's rotation axis, and $C_{11}$, $C_{33}, C_{44}, C_{12}, C_{13}$ the elastic constants for a cylindrically anisotropic (or transversely isotropic) medium:

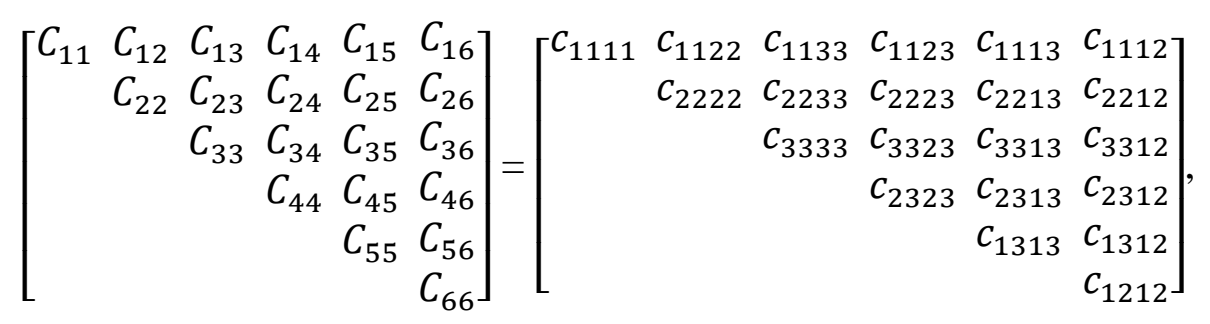

639 in which $\left(c_{i j k l}\right) i, j, k, l=1,2,3$ represents the tensor for elastic constants. We calculate the moduli for 640 models with different crystal alignments using the coordinate transformation law (Auld, 1990):

$$
c_{\text {mnop }}=a_{m i} a_{n j} a_{o k} a_{p l} c_{i j k l}(i, j, k, l, m, n, o, p=1,2,3),
$$


642 in which $\left(a_{i j}\right)$ is the matrix for coordinate transformation. The elastic constants for different IC iron

643 models are in Supplementary Table 1. We calculate the averaged shear wave speed following Lin et al., 644 (2010) and Mao et al., (2008):

$$
\frac{2}{V_{S}^{3}}=\frac{1}{V_{S 1}^{3}}+\frac{1}{V_{S 2}^{3}}
$$

646 in which $V_{S 1}$ and $V_{S 2}$ are speed of $\mathrm{J}$ waves of the two polarizations. Calculation results for different models 647 are in Figure S9.

Synthetic experiments for IC shear wave stacks at different $\varphi$ angles

651 We perform synthetic experiments to show how IC shear-wave anisotropy can decrease the amplitude of 652 I2-J stacks when $\varphi$ approaches $0^{\circ}$. We set an IC model made of a single crystal of hcp structure (Figure 4 653 and Table S1). We then calculate the relative travel times and produce synthetic waveforms for $\mathrm{J}$ waves 654 propagating in different directions. We do not consider the compressional-wave anisotropy in the IC, 655 because the compressional waves (I waves) contributing to the I2-J feature sample the topmost IC at 656 maximal depths less than $10 \mathrm{~km}$ below the ICB (Figure S11c). Apart from that, the thin layer (thickness 657 of a few tens of kilometers) in the upper IC was suggested to be quasi-isotropic or weakly anisotropic (less 658 than 1\%) (e.g., Shearer, 1994; Song \& Helmberger, 1995). In the calculation, we take ellipticity into 659 account. We use Ricker wavelets to represent synthetic waveforms by shifting them with respect to the 660 calculated travel times. They are bandpass filtered in the interval 15-50 s, which is the dominant frequency 661 range for coda-correlation wavefield. Then, for each angle $\varphi$, we calculate the composition of $\mathrm{J}$ waves for 662 varied angle $\xi$ (Figures S10a-b). The composition is described by the probability density function (PDF) 663 with respect to angle $\xi$ (the angle between $\mathrm{J}$ wave ray paths and the rotation axis). Finally, we calculate 664 waveform stacks (Figures S10d,f,h) based on the synthetics and the PDFs. The stacking is weighted by 665 the PDFs. We perform the tests for J waves with polarization parallel to meridians (Figures S10c-d) and 666 the equator (Figures S10E-F), and the averaged J waves (Figures S10g-h). 


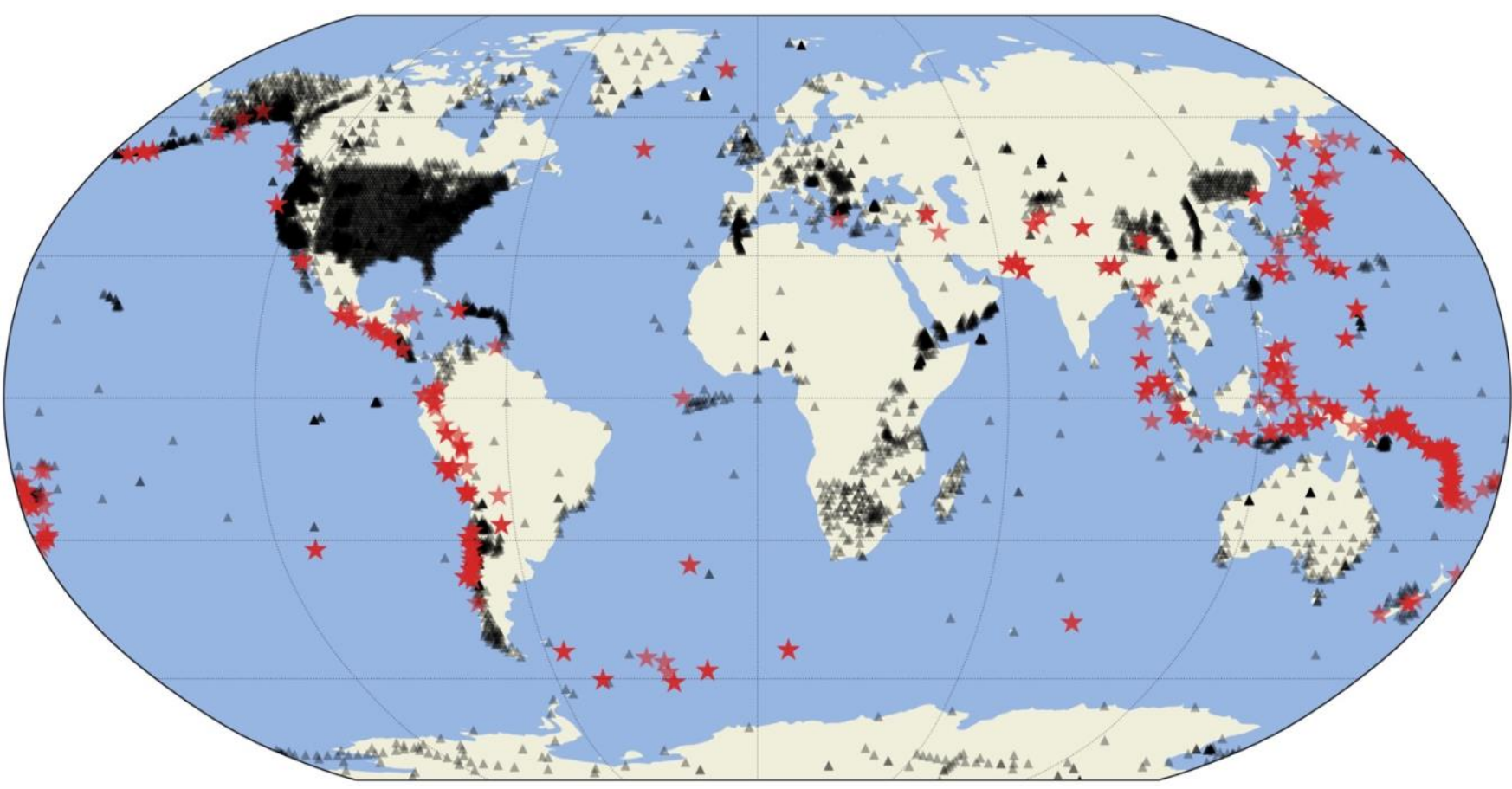

671 Figure S1. Global distributions of events (red stars) and stations (black triangles) used in this study.

$672 \mathrm{Mw} \geq 6.8$ earthquakes in 2010-2019 from the National Earthquake Information Center (NEIC) catalog

673 (https://pubs.er.usgs.gov/publication/70016044) are used, as listed in Table S2. Seismic waveform data

674 are retrieved from IRIS DMC (https://ds.iris.edu/ds/nodes/dmc/data). Stations are from networks

675 identified according to FDSN (https://www.fdsn.org/networks/): 1P, 2H, 2K, 3J, 4F, 5A, 6A, 6D, 6E,

676 7A, 7C, 8A, 9C, 9D, AC, AD, AE, AF, AI, AK, AT, AU, AV, AZ, BC, BE, BK, BL, BN, BX, C, C1,

$677 \mathrm{CA}, \mathrm{CB}, \mathrm{CC}, \mathrm{CH}, \mathrm{CI}, \mathrm{CK}, \mathrm{CM}, \mathrm{CN}, \mathrm{CU}, \mathrm{CZ}, \mathrm{DK}, \mathrm{DR}, \mathrm{EI}, \mathrm{EP}, \mathrm{ER}, \mathrm{ES}, \mathrm{EV}, \mathrm{G}, \mathrm{GB}, \mathrm{GE}, \mathrm{GG}, \mathrm{GR}, \mathrm{GS}$,

678 GT, HK, HL, HT, HV, HW, IC, II, IM, IO, IP, IU, IW, JP, KC, KG, KN, KO, KP, KR, KS, KW, KZ,

679 LB, LD, LI, LM, LX, MB, MC, MG, MI, MM, MN, MS, MU, MX, MY, N4, NA, NE, NJ, NK, NL,

680 NM, NN, NO, NR, NU, OE, OH, OK, ON, OO, OV, PB, PE, PI, PL, PM, PN, PO, PP, PR, PS, PT, PY,

681 RB, RM, RO, RV, S1, SB, SC, SL, SS, SV, TA, TC, TM, TR, TS, TT, TW, TX, UK, UO, US, UU, UW,

682 VE, WC, WI, WM, WY, X1, X3, X5, X9, XB, XD, XE, XF, XH, XI, XN, XP, XR, XS, XU, XV, XW,

683 XZ, Y2, Y5, Y6, YB, YD, YE, YF, YG, YH, YL, YM, YN, YP, YS, YT, YW, YY, YZ, Z1, Z2, Z4, Z5,

684 Z6, ZC, ZM, ZN, ZP, ZT, ZV. 
686

(a)

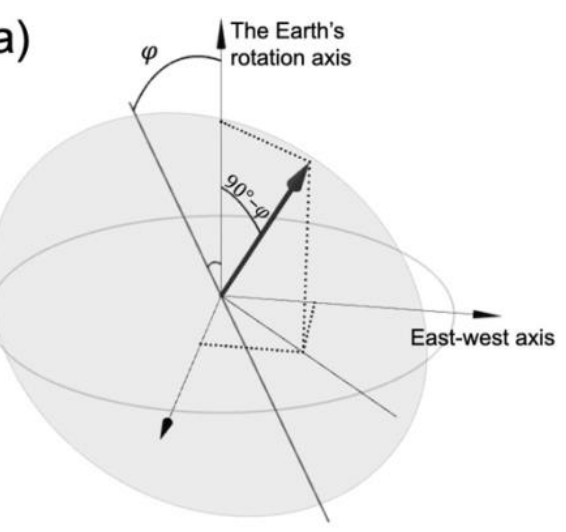

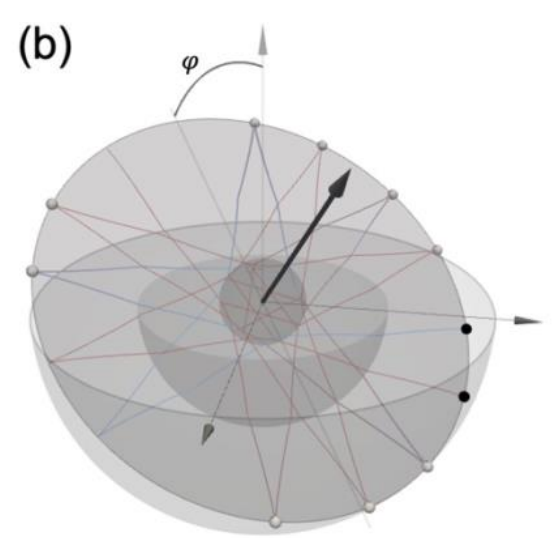

688 Figure S2. (a) A great-circle plane is described by its normal vector (black arrow) in three dimensions.

$689 \varphi$ is the angle between the great-circle plane and the Earth's rotation axis. $90^{\circ}-\varphi$ is the angle between 690 the normal vector and the rotation axis. (b) A great-circle plane passing through two receivers (black 691 spheres) and events (white spheres). (c) Another great-circle plane with the same angle $\varphi$ as in (b) but 692 with a different normal vector. The receiver pairs are binned in ranges with respect to $\varphi$ (Figure S3) to 693 form nine I2-J stacks (Figure S4). 
(a)

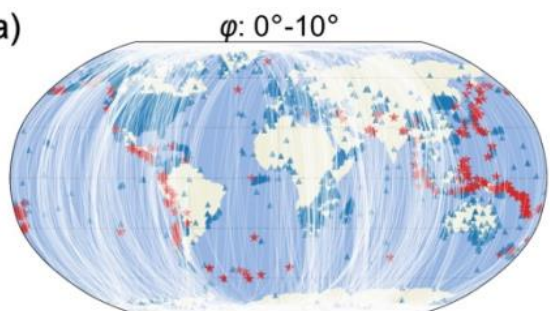

(d)

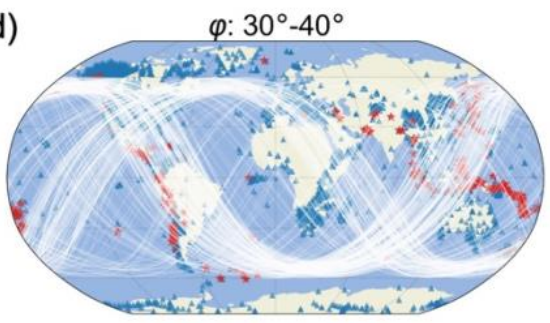

(g)

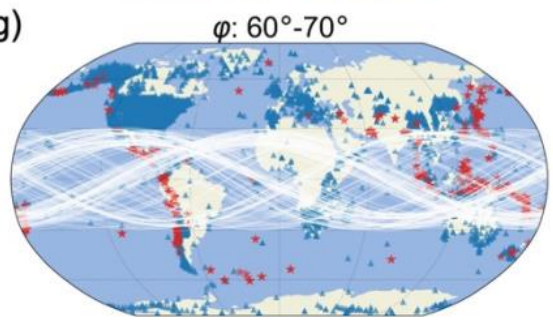

(b)

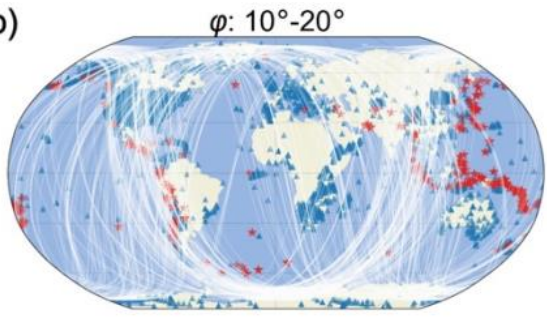

(e)

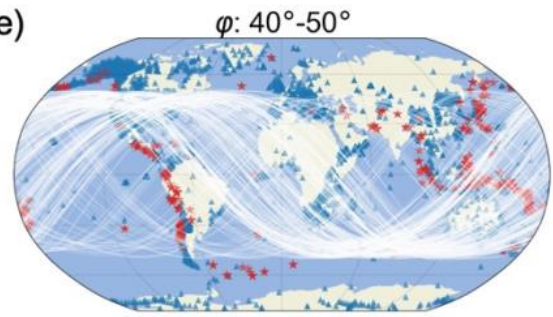

(h)

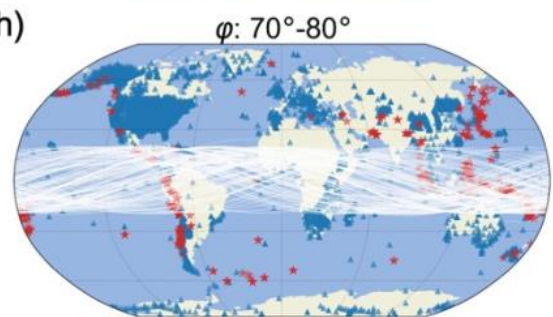

(c)

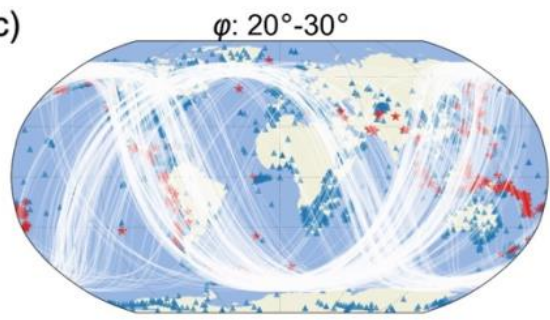

(f)

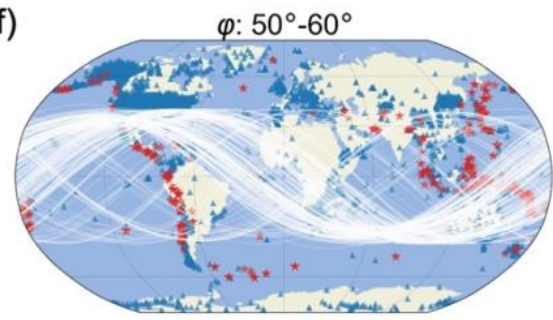

(i)

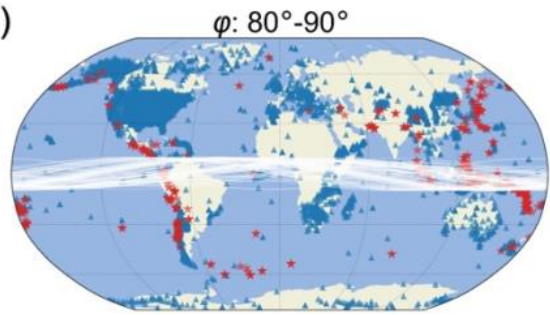

696 Figure S3. Great-circle planes (white lines) for different angle $\varphi$ ranges: (a) $0^{\circ}-10^{\circ}$, (b) $10^{\circ}-20^{\circ}$, (c) $20^{\circ}-$ $69730^{\circ}$, (d) $30^{\circ}-40^{\circ}$, (e) $40^{\circ}-50^{\circ}$, (f) $50^{\circ}-60^{\circ}$, (g) $60^{\circ}-70^{\circ}$, (h) $70^{\circ}-80^{\circ}$, (i) $80^{\circ}-90^{\circ}$. The angle $\varphi$ is defined in 698 Figure 2 and Figure S2. See Methods for grouping of correlograms for the angle $\varphi$. We plot randomly 699 downsampled great-circle planes instead of all planes to avoid intense overlapping. 


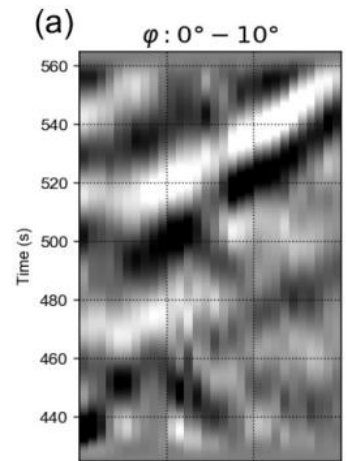

(b) $\varphi: 10^{\circ}-20^{\circ}$
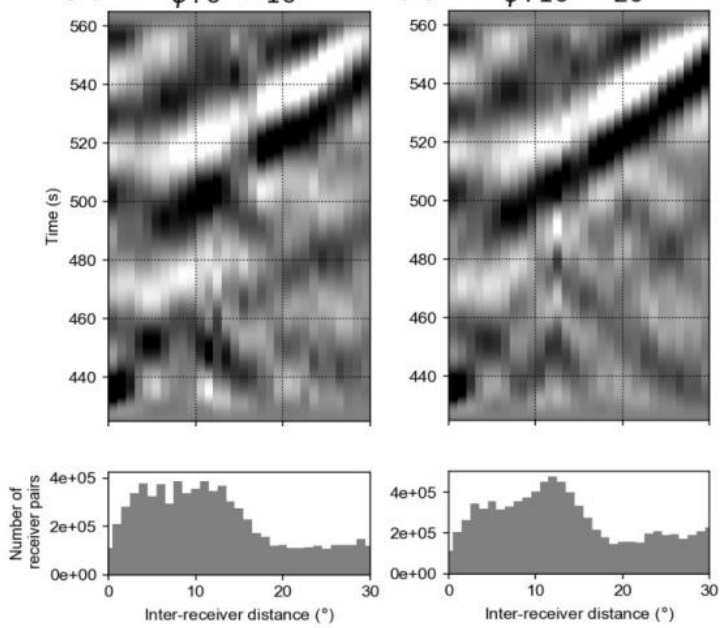

(f)
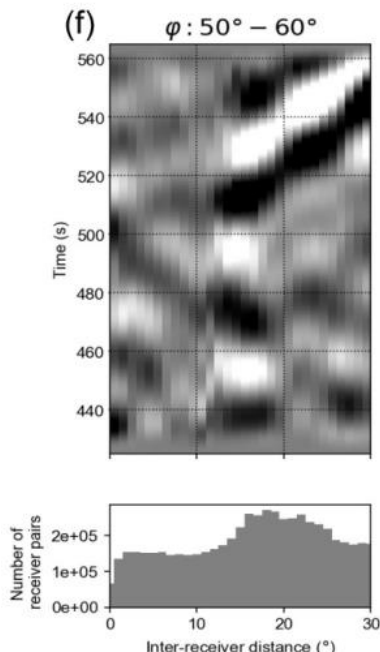

(c)

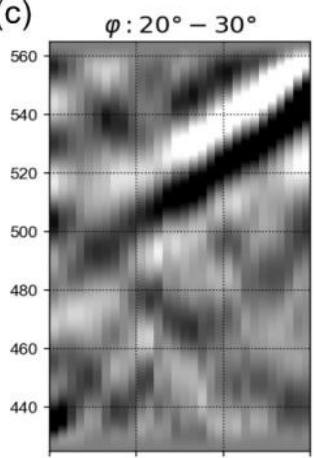

(d)

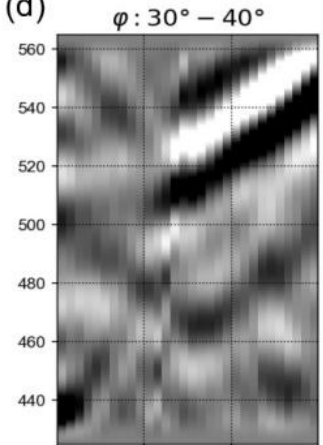

(e) $\quad \varphi: 40^{\circ}-50^{\circ}$
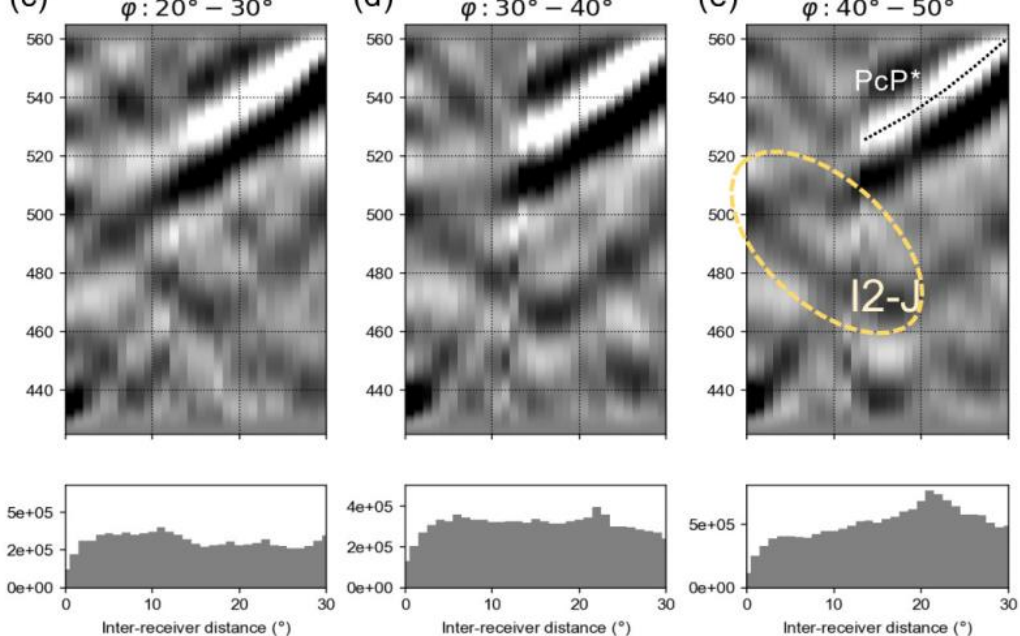

(g) $\quad \varphi: 60^{\circ}-70^{\circ} \quad$ (h)

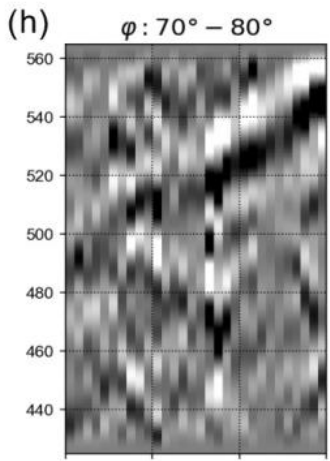

(i)
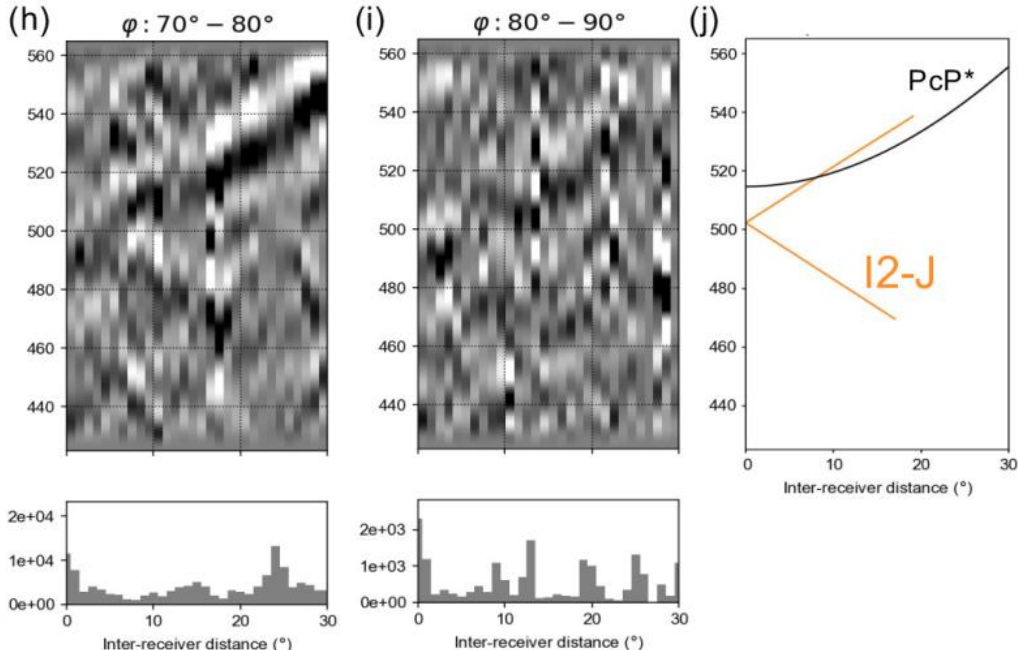

Figure S4. Earthquake Coda correlograms and histograms of receiver pair numbers for nine angle $\varphi$ $80^{\circ}$, (i) $80^{\circ}-90^{\circ} . \varphi$ is the angle between a great-circle plane and the Earth's rotation axis, as defined in Figures 2 and S2. The orange ellipse (shown only in (e) to avoid overlapping with the correlogram features for the sake of clarity) indicates the I2-J cusp sensitive to the IC shear-wave speed. The feature PcP*, sensitive to mantle structure, CMB topography, and Earth's ellipticity, is indicated by the black dotted line. Positive amplitudes are in white, and negative amplitudes are in black shades. The intensity of the black or white indicates the amplitude strength. (j) Theoretical I2-J and PcP* time curves based on Tkalčić \& Phạm (2018). 
(a)

716

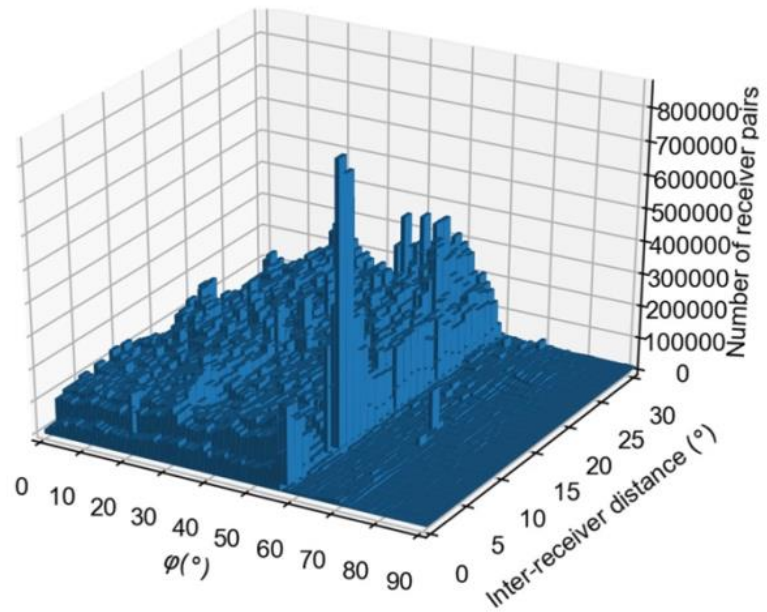

(b)

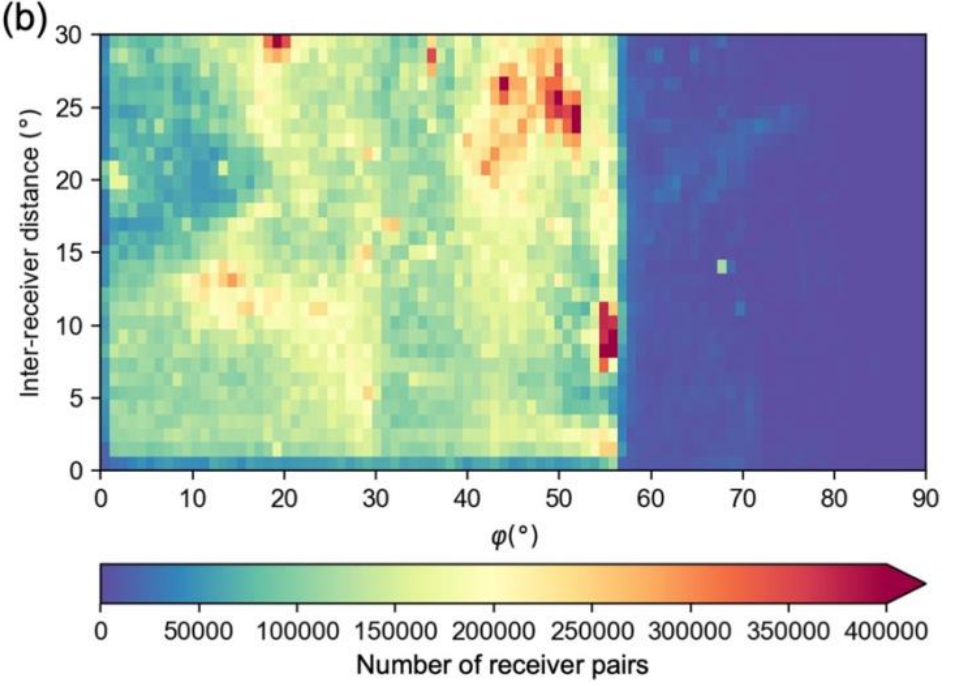

717 Figure S5. (a) 3D histogram of the number of receiver pairs relative to angle $\varphi$ (as defined in Figs. 2

718 and S2) and inter-receiver distance. See the Methods section for the cliff-like change for $\varphi \sim 60^{\circ}$ and the 719 column spike for $\varphi \sim 69^{\circ}$ and inter-receiver distance $\sim 15^{\circ}$. (b) Isogram of the 3D histogram in (a). 
(a)

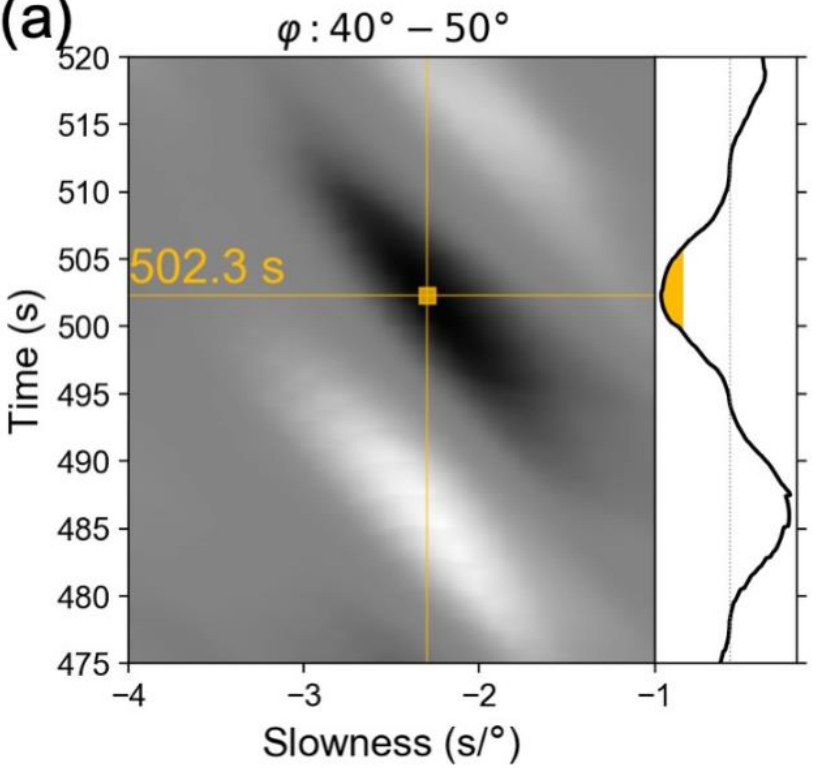

(c)

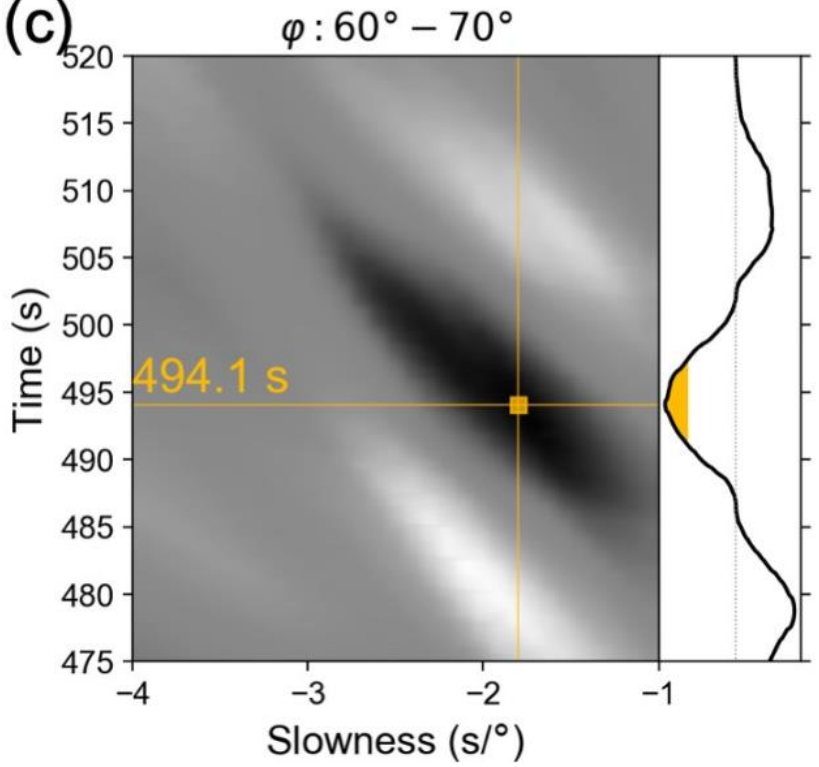

(b)

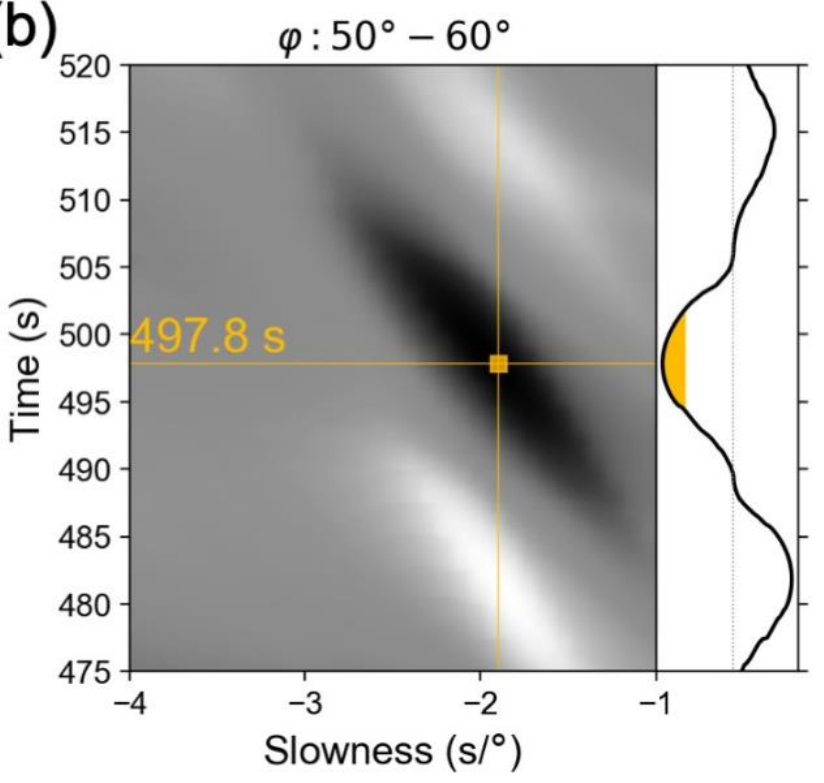

(d)

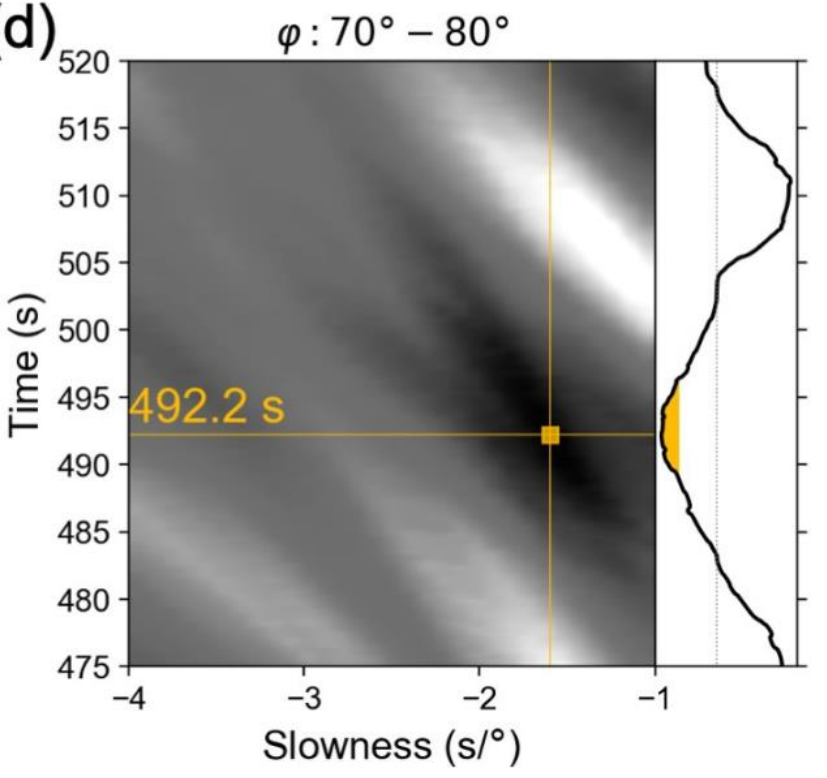

722 Figure S6. I2-J slant-stacks for four angle $\varphi$ ranges: (a) $40^{\circ}-50^{\circ}$, (b) $50^{\circ}-60^{\circ}$, (c) $60^{\circ}-70^{\circ}$, (d) $70^{\circ}-80^{\circ}$.

723 The yellow dots correspond to the lower branch of the I2-J cusp. $\varphi$ is the angle between a great-circle

724 plane and the Earth's rotation axis, as defined in Figures 2 and S2. Each slant stack is normalized with 725 respect to the maximal amplitude. Details of the slant-stack method are in Methods section. 

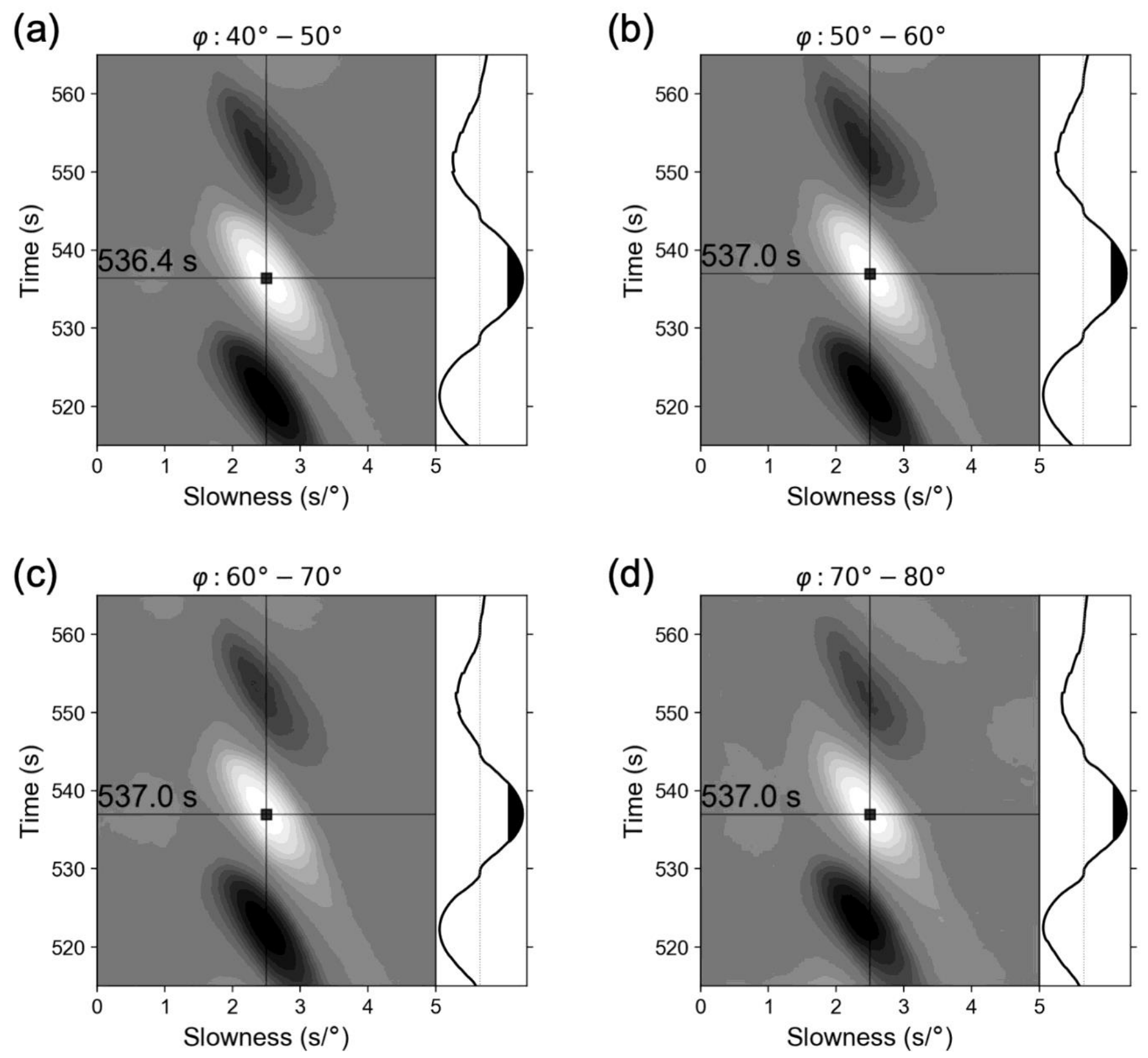

728 Figure S7. PcP* slant-stacks for four angle $\varphi$ ranges: (a) $40^{\circ}-50^{\circ}$, (b) $50^{\circ}-60^{\circ}$, (c) $60^{\circ}-70^{\circ}$, (d) $70^{\circ}-80^{\circ}$.

729 The $\varphi$ is the angle between a great-circle plane and the Earth's rotation axis, as defined in Figures 2 and

730 S2. Each slant stack is normalized with respect to the maximal amplitude. Details of the slant-stack 731 method are in Methods section. 
(a)

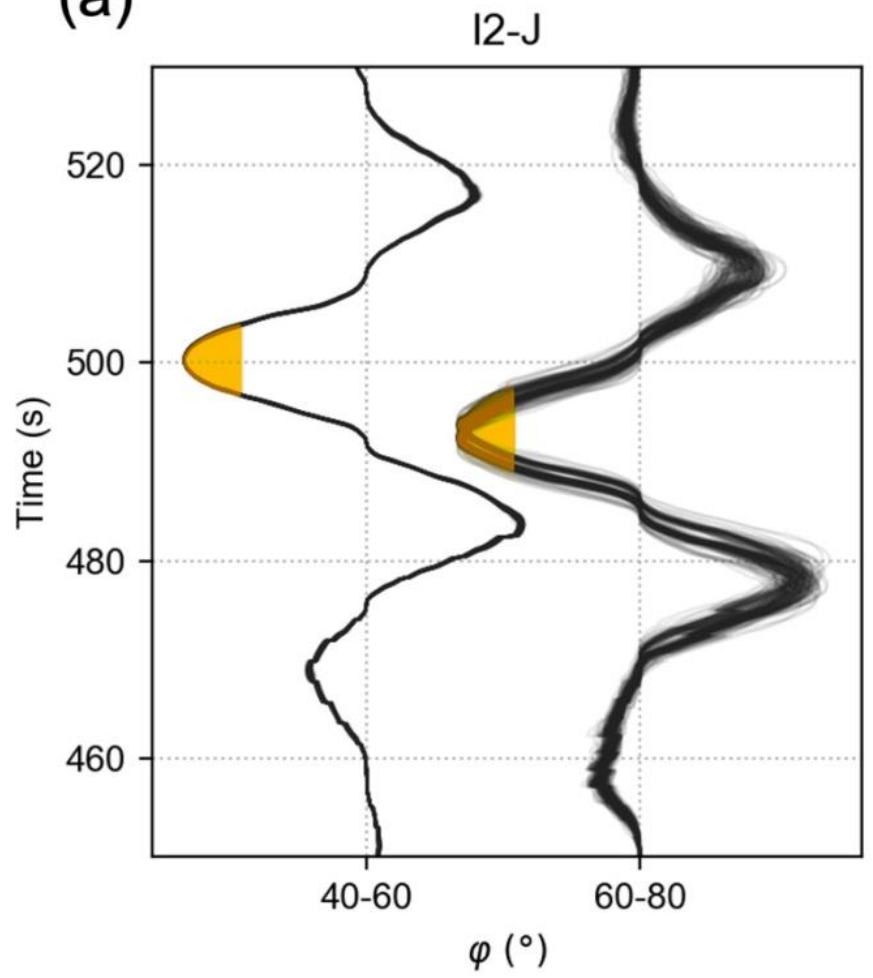

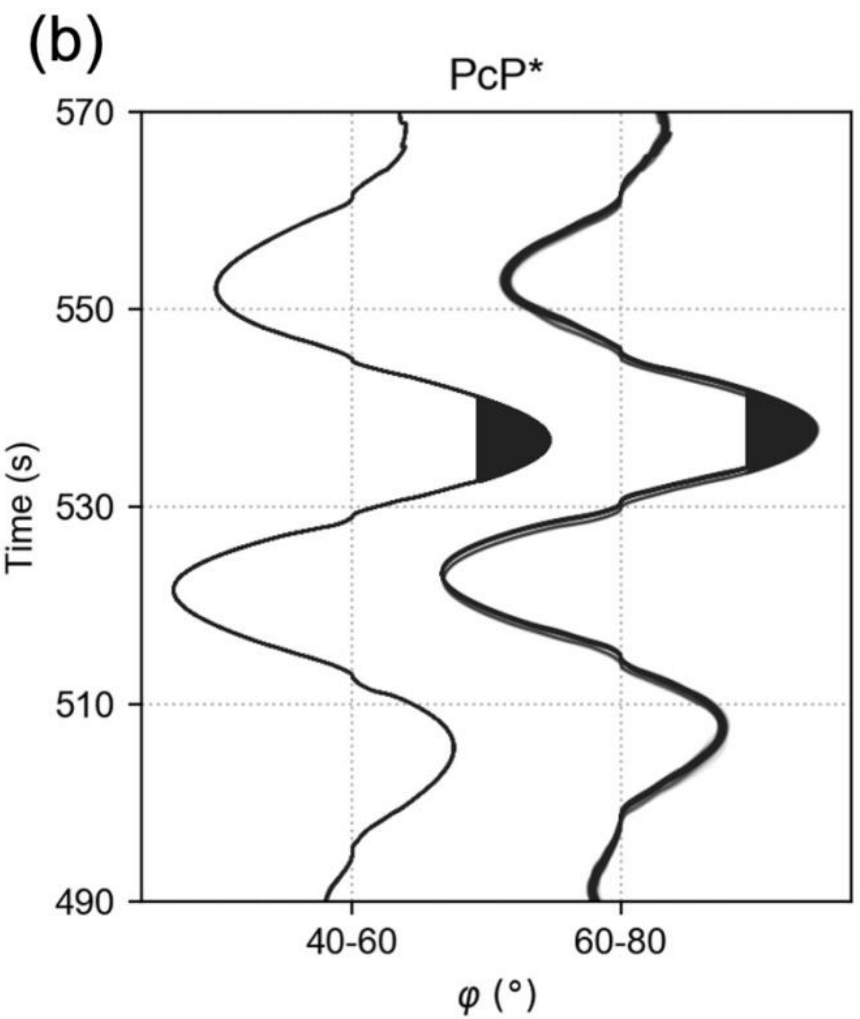

734 Figure S8. Bootstrap experiments for travel time measurements of I2-J (a) and PcP* (b). See Methods 735 section for details of bootstrap experiments. Waveform stacks for (a) I2-J and (b) PcP* correlogram 736 features are extracted from the slant-stacks. Each waveform stack (represented by black lines)

737 corresponds to a single random resampling. Colored areas correspond to stack amplitudes greater than $73880 \%$ of the maximum. 
(a)

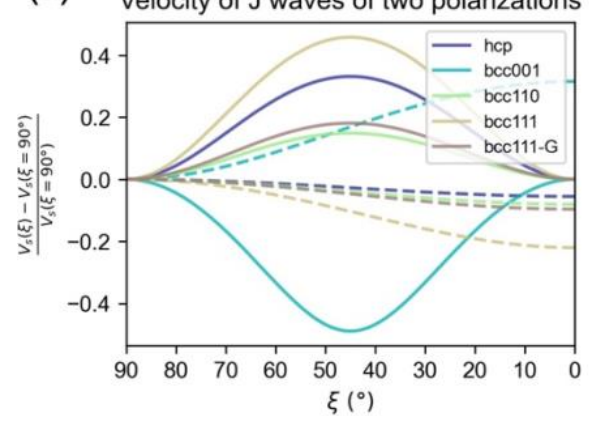

(b)

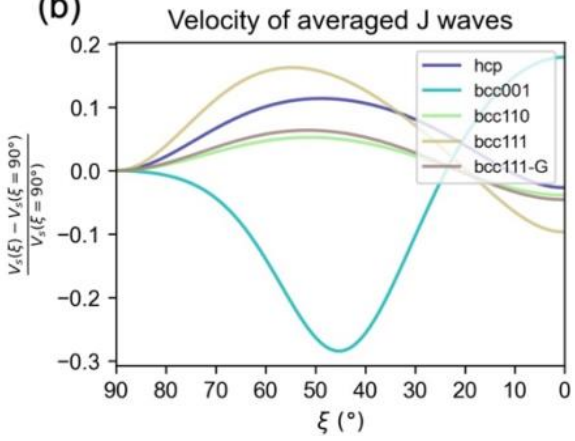

(c)

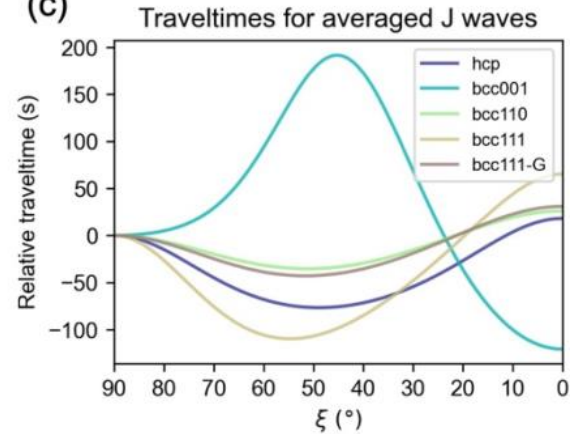

Figure S9. (a) Fractional J-wave speed (with respect to the speed in the equatorial plane) for two

742 polarizations as a function of the angle $\xi$ defined in Figure 4 for a cylindrically anisotropic inner core.

743 The solid lines represent the $\mathrm{J}$ waves with the polarization parallel to meridians, and the dash lines

744 represent the $\mathrm{J}$ waves with the polarization parallel to the equator. The colors represent different iron

745 crystal models, as listed in Table S1. The 3D iron crystal models of hcp and bcc are illustrated in Figure

746 4. (b) Same as (a) but for averaged (both polarizations) J waves. (c) Relative travel time for averaged J

747 waves traveling along the IC diameters. See Methods section for calculations of J-wave speed given

748 elastic properties of an iron crystal model. 
(a)

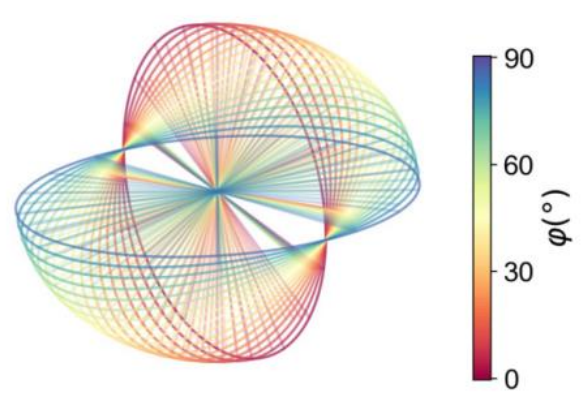

(c)

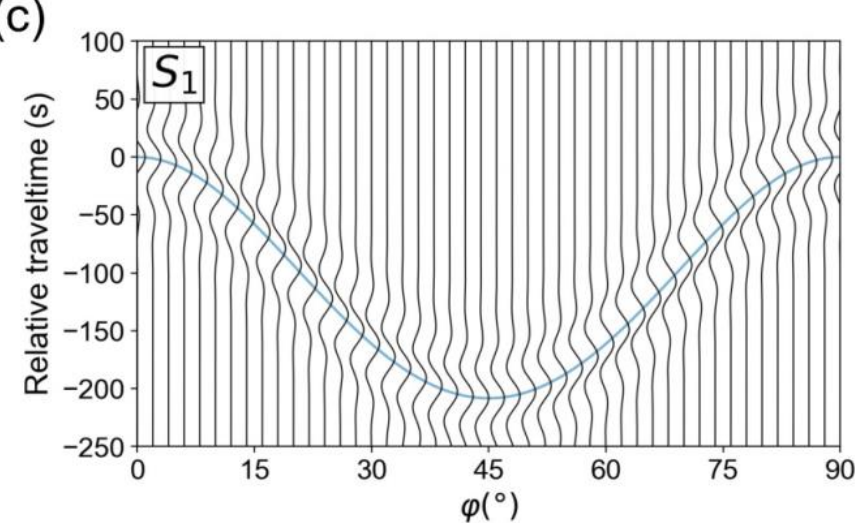

(e)

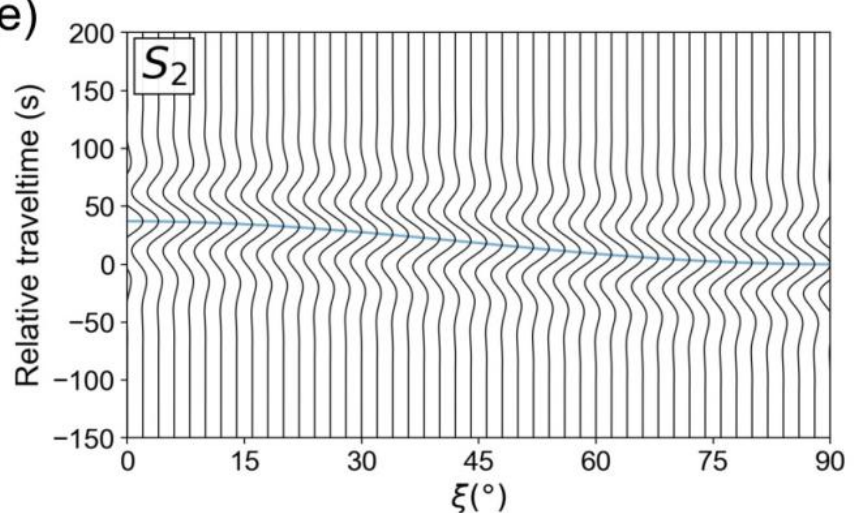

(g)

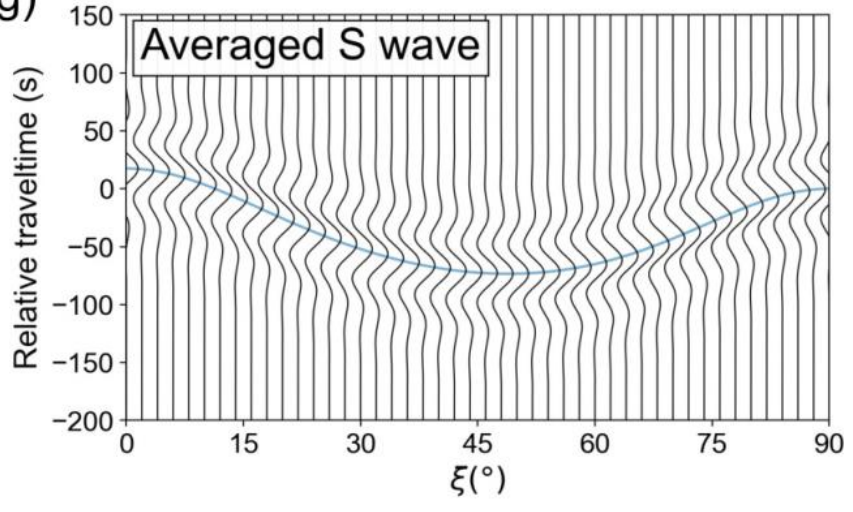

(b)

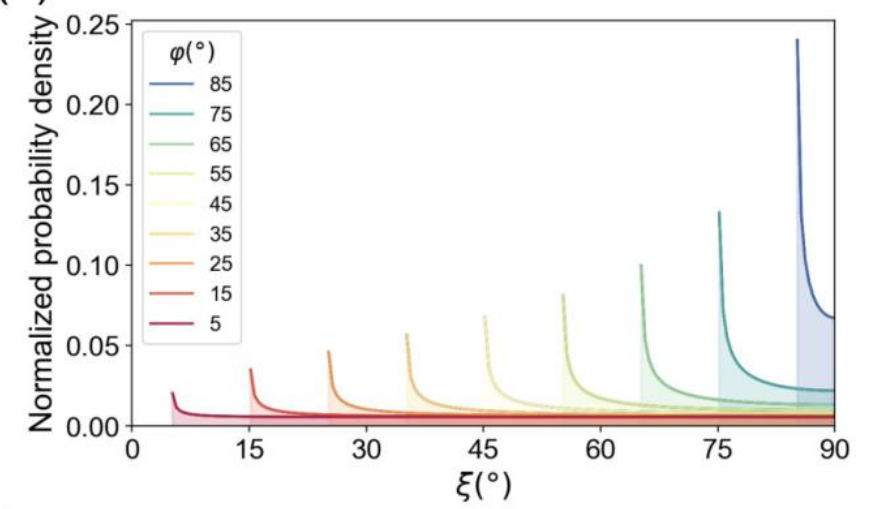

(d)

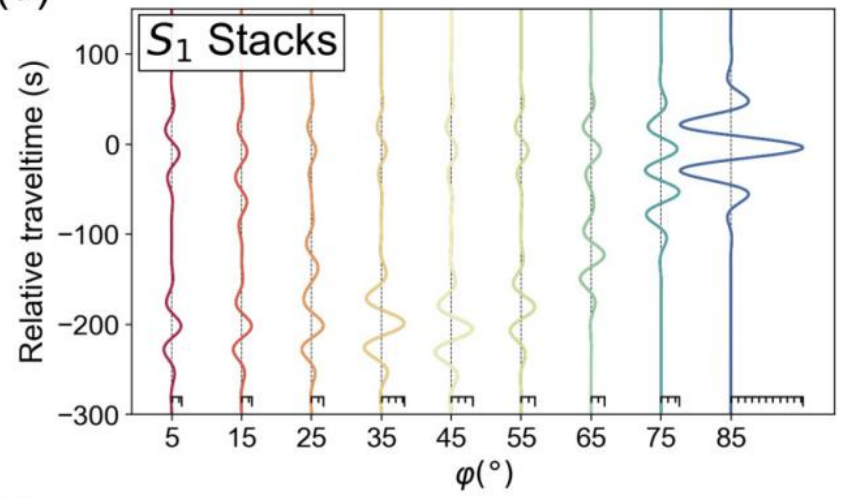

(f)

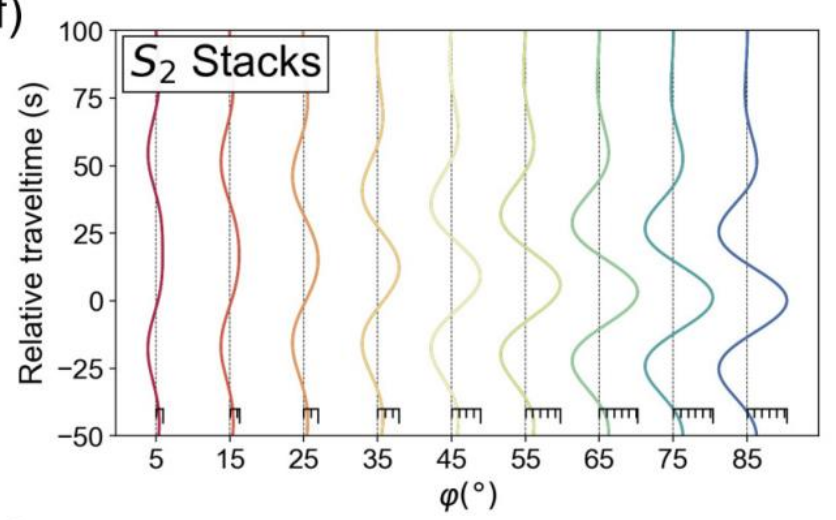

(h)

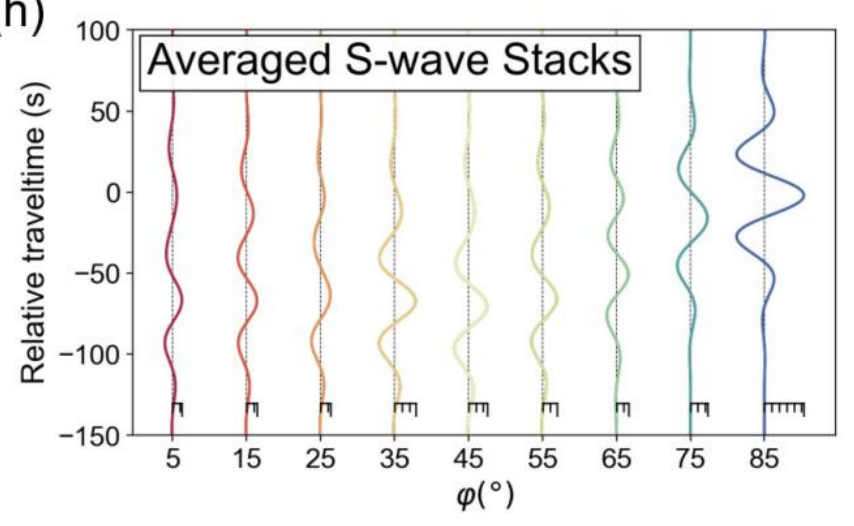


751 Figure S10. Synthetic tests for I2-J stacks at different angles $\varphi$ given an anisotropic inner core. (a) Inner752 core shear-wave ray paths inside great-circle planes for varied angles $\varphi$ shown in different colors. The $\varphi$ 753 is the angle between a great-circle plane and the Earth's rotation axis, as defined in Figures 2 and S2. (b) 754 The probability density of inner-core shear-wave angle $\xi$ for great-circle planes at different $\varphi$. The $\xi$ is 755 the angle between inner-core shear wave ray paths and the rotation axis. The shaded areas indicate the 756 integrals of the probability density functions that correspond to the weighting-factors for computing the 757 synthetic waveform stacks for different $\varphi$. (c) Synthetic waveforms of inner-core shear wave $S_{1}$ that 758 propagate at different angles $\xi$. The $\mathrm{S}_{1}$ has the polarization parallel the meridians and passes through the 759 Earth's center. The relative travel time curve (blue line) is based on the hcp iron model in Figure 4 and 760 Table S1 and corrected for the Earth's ellipticity. Other iron crystal models have similar behavior. We use 761 Ricker wavelets bandpass-filtered in the 15-50 s range, which is the dominant frequency range of the coda762 correlation wavefield. (d) Stacked $\mathrm{S}_{1}$ waveforms for different angles $\varphi$ based on the probability density of $763 \xi(\mathrm{b})$. The scale bars indicate the relative amplitude difference for waveform stacks for different angles $\varphi$. 764 (e) Similar to (c) but for inner-core shear waves $S_{2}$ that have the polarization parallel to the equator. (f) 765 Similar to (d) but for $\mathrm{S}_{2}$ wave stacks. (g) Similar to (c) but for averaged shear waves in the inner core. The 766 average uses the expression $2 / V_{S}=1 / V_{S 1}+1 / V_{S 2}$ (Lin et al., 2010; Mao et al., 2008) . (h) Similar to (d) 767 but for averaged S-wave stacks. 
(a)

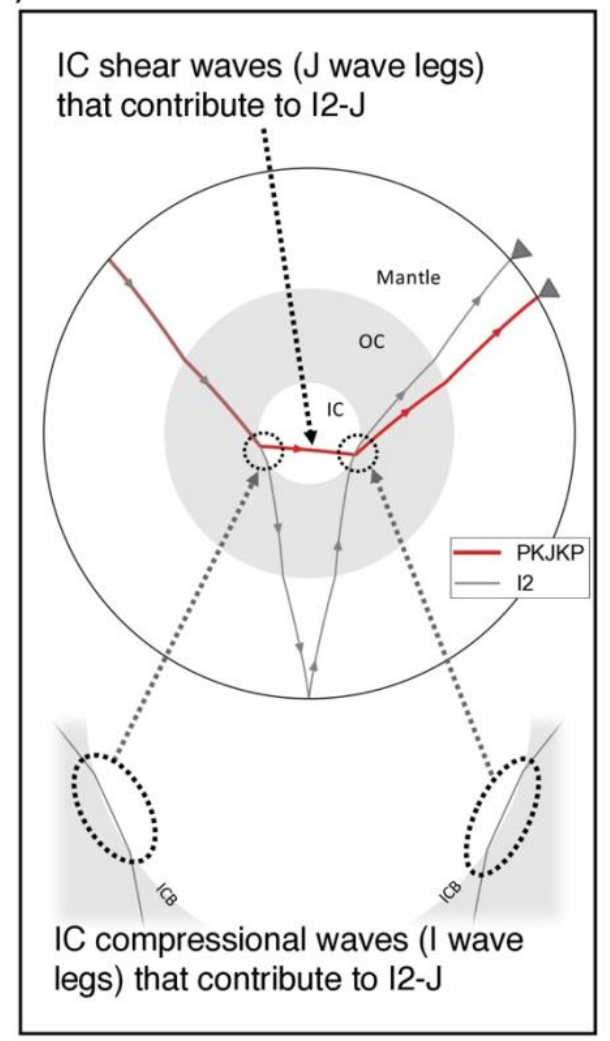

(b)

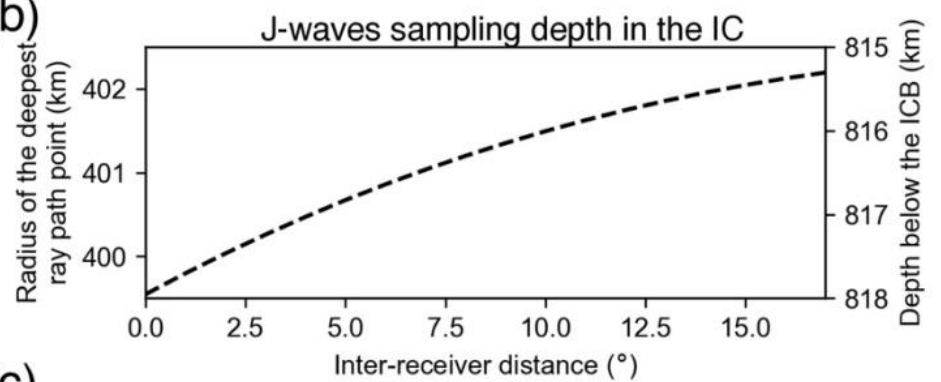

(c)

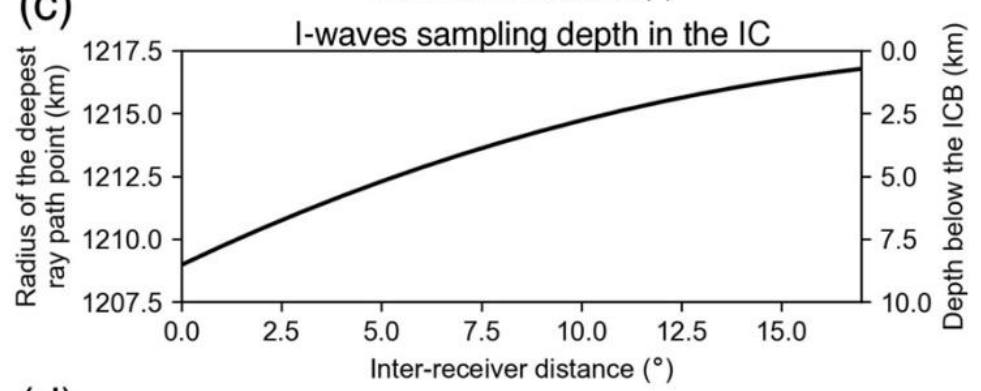

(d)

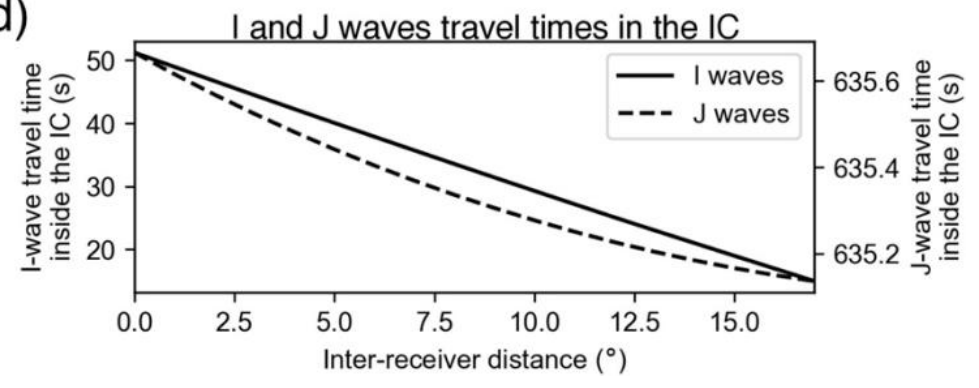

Figure S11. A sampling of the IC for shear waves and compressional waves that contribute to the codacorrelation feature I2-J. (a) Diagram for I2-J ray path. The I2-J is formed by the cross-correlation between

771 PKIKPPKIKP (grey line) and PKJKP (red line). The IC shear waves (J waves) that contribute to I2-J are 772 indicated with a dashed arrow. The insets show the IC compressional waves (I waves) that contribute to 773 I2-J. (b) Radius/depth of the deepest ray path point for J waves contributing to I2-J as a function of inter774 receiver distance. We use the reference model ak135 (Kennett et al., 1995) for computing the ray path. 775 We choose the lower branch of the I2-J to analyze the time variations (see Methods section in the 776 supporting information). (c) The same as (b) but for I waves contributing to I2-J. (d) The total travel time 777 for J and I (double legs) waves that contribute to the I2-J. 
Table S1. Elastic properties of inner-core iron crystal models used in this study. The elastic properties

785 for hep and bcc001 are provided by Mattesini et al. (2010), Romanowicz et al. (2016), and Vočadlo et al.

786 (2009). We compute the elastic properties for bcc110 and bcc111 via rotating the bcc001 model

787 (Methods). bcc111-G corresponds to an imperfect alignment of the bcc111 crystal (see main text). The 788 anisotropy of S waves is $A n(\%)=200\left(S_{\max }-S_{\min }\right) /\left(S_{\max }+S_{\min }\right)$.

\begin{tabular}{|c|c|c|c|c|c|c|c|}
\hline Iron model & $\rho(\mathrm{g} / \mathrm{cm} 3)$ & $c 11(\mathrm{GPa})$ & $c 33$ & $c 44$ & $c 12$ & $c 13$ & Anisotropy of S $(\%)$ \\
\hline hcp & 13.543 & 1700 & 1769 & 200 & 1252 & 1025 & 13.5 \\
\hline bcc001 & 13.559 & 1715 & 1561 & 365 & 1293 & 1448 & 48.9 \\
\hline bcc110 & 13.559 & 1831 & 1870 & 211 & 1332 & 1293 & 9.0 \\
\hline bcc111 & 13.559 & 1870 & 1973 & 159 & 1345 & 1242 & 25.1 \\
\hline bcc111-G & 13.559 & 1836 & 1882 & 205 & 1334 & 1287 & 10.8 \\
\hline
\end{tabular}

Table S2. List of events used in this study.

\begin{tabular}{|c|c|c|c|c|}
\hline \multicolumn{5}{|c|}{ Table S2. List of events used in this study } \\
\hline Longitude $\left({ }^{\circ}\right)$ & Latitude $\left({ }^{\circ}\right)$ & Depth $(\mathrm{km})$ & Magnitude $(\mathrm{Mw})$ & Origin Time \\
\hline 125.17 & 6.7 & 18 & 6.8 & 2019-12-15T06:11:51 \\
\hline 126.42 & 1.62 & 33 & 7.1 & 2019-11-14T16:17:40 \\
\hline 104.79 & -7.28 & 49 & 6.9 & $2019-08-02 \mathrm{~T} 12: 03: 27$ \\
\hline-72.31 & -34.24 & 25 & 6.8 & $2019-08-01 \mathrm{~T} 18: 28: 07$ \\
\hline 128.03 & -0.59 & 19 & 7.2 & $2019-07-14 \mathrm{~T} 09: 10: 51$ \\
\hline 126.19 & 0.51 & 35 & 6.9 & $2019-07-07 \mathrm{~T} 15: 08: 40$ \\
\hline 129.17 & -6.41 & 212 & 7.3 & $2019-06-24 \mathrm{~T} 02: 53: 39$ \\
\hline-178.1 & -30.64 & 46 & 7.3 & $2019-06-15 \mathrm{~T} 22: 55: 04$ \\
\hline-75.27 & -5.81 & 123 & 8 & $2019-05-26 \mathrm{~T} 07: 41: 15$ \\
\hline 152.6 & -4.05 & 10 & 7.6 & $2019-05-14 \mathrm{~T} 12: 58: 25$ \\
\hline 146.45 & -6.97 & 146 & 7.1 & $2019-05-06 \mathrm{~T} 21: 19: 37$ \\
\hline 122.58 & -1.81 & 15 & 6.8 & $2019-04-12 \mathrm{~T} 11: 40: 49$ \\
\hline-70.16 & -14.71 & 267 & 7 & $2019-03-01 \mathrm{~T} 08: 50: 42$ \\
\hline-77.05 & -2.19 & 145 & 7.5 & $2019-02-22 \mathrm{~T} 10: 17: 23$ \\
\hline-71.59 & -8.14 & 570 & 6.8 & $2019-01-05 \mathrm{~T} 19: 25: 38$ \\
\hline 126.92 & 5.9 & 60 & 7 & $2018-12-29 \mathrm{~T} 03: 39: 09$ \\
\hline 164.7 & 55.1 & 17 & 7.3 & $2018-12-20 \mathrm{~T} 17: 01: 55$ \\
\hline-26.39 & -58.55 & 133 & 7.1 & $2018-12-11 \mathrm{~T} 02: 26: 29$ \\
\hline
\end{tabular}




\begin{tabular}{|c|c|c|c|c|}
\hline 169.43 & -21.95 & 10 & 7.5 & $2018-12-05 \mathrm{~T} 04: 18: 08$ \\
\hline-149.96 & 61.35 & 47 & 7.1 & $2018-11-30 \mathrm{~T} 17: 29: 29$ \\
\hline-178.93 & -17.87 & 540 & 6.8 & $2018-11-18 \mathrm{~T} 20: 25: 46$ \\
\hline 20.56 & 37.52 & 14 & 6.8 & $2018-10-25 \mathrm{~T} 22: 54: 52$ \\
\hline-129.29 & 49.34 & 10 & 6.8 & $2018-10-22 \mathrm{~T} 06: 16: 26$ \\
\hline 151.21 & -5.7 & 39 & 7 & $2018-10-10 \mathrm{~T} 20: 48: 20$ \\
\hline 119.85 & -0.26 & 20 & 7.5 & $2018-09-28 \mathrm{~T} 10: 02: 45$ \\
\hline-179.37 & -31.75 & 115 & 6.9 & $2018-09-10 \mathrm{~T} 04: 19: 02$ \\
\hline 179.35 & -18.47 & 671 & 7.9 & $2018-09-06 \mathrm{~T} 15: 49: 18$ \\
\hline 170.13 & -22.03 & 21 & 7.1 & $2018-08-29 \mathrm{~T} 03: 51: 56$ \\
\hline-70.83 & -11.04 & 630 & 7.1 & $2018-08-24 \mathrm{~T} 09: 04: 08$ \\
\hline-62.9 & 10.77 & 147 & 7.3 & $2018-08-21 \mathrm{~T} 21: 31: 47$ \\
\hline 116.63 & -8.32 & 21 & 6.9 & $2018-08-19 \mathrm{~T} 14: 56: 27$ \\
\hline-178.15 & -18.11 & 600 & 8.2 & $2018-08-19 \mathrm{~T} 00: 19: 40$ \\
\hline 116.44 & -8.26 & 34 & 6.9 & $2018-08-05 \mathrm{~T} 11: 46: 38$ \\
\hline-63.01 & -20.66 & 559 & 6.8 & $2018-04-02 \mathrm{~T} 13: 40: 34$ \\
\hline 151.5 & -5.53 & 35 & 6.9 & $2018-03-29 \mathrm{~T} 21: 25: 36$ \\
\hline 153.2 & -4.38 & 23 & 6.8 & $2018-03-08 \mathrm{~T} 17: 39: 51$ \\
\hline 142.75 & -6.07 & 25 & 7.5 & $2018-02-25 \mathrm{~T} 17: 44: 44$ \\
\hline-97.98 & 16.39 & 22 & 7.2 & $2018-02-16 \mathrm{~T} 23: 39: 39$ \\
\hline-149.17 & 56 & 14 & 7.9 & $2018-01-23 \mathrm{~T} 09: 31: 40$ \\
\hline-74.71 & -15.77 & 39 & 7.1 & $2018-01-14 \mathrm{~T} 09: 18: 45$ \\
\hline-83.52 & 17.48 & 19 & 7.5 & $2018-01-10 \mathrm{~T} 02: 51: 33$ \\
\hline 168.67 & -21.32 & 10 & 7 & $2017-11-19 \mathrm{~T} 22: 43: 29$ \\
\hline 45.96 & 34.91 & 19 & 7.3 & $2017-11-12 \mathrm{~T} 18: 18: 17$ \\
\hline-173.17 & -15.32 & 10 & 6.8 & $2017-11-04 \mathrm{~T} 09: 00: 19$ \\
\hline-98.49 & 18.55 & 48 & 7.1 & $2017-09-19 \mathrm{~T} 18: 14: 38$ \\
\hline-93.9 & 15.02 & 47 & 8.2 & $2017-09-08 \mathrm{~T} 04: 49: 19$ \\
\hline 168.86 & 54.44 & 10 & 7.7 & $2017-07-17 \mathrm{~T} 23: 34: 13$ \\
\hline-90.97 & 13.72 & 38 & 6.8 & $2017-06-22 \mathrm{~T} 12: 31: 03$ \\
\hline-92.01 & 14.91 & 93 & 6.9 & $2017-06-14 \mathrm{~T} 07: 29: 04$ \\
\hline 167.38 & -14.59 & 169 & 6.8 & $2017-05-09 \mathrm{~T} 13: 52: 10$ \\
\hline 125.07 & 5.5 & 26 & 6.9 & $2017-04-28 \mathrm{~T} 20: 23: 17$ \\
\hline-72.06 & -33.04 & 28 & 6.9 & $2017-04-24 \mathrm{~T} 21: 38: 30$ \\
\hline-178.8 & -23.26 & 414 & 6.9 & $2017-02-24 \mathrm{~T} 17: 28: 44$ \\
\hline 155.17 & -6.25 & 135 & 7.9 & $2017-01-22 \mathrm{~T} 04: 30: 22$ \\
\hline 122.62 & 4.48 & 627 & 7.3 & $2017-01-10 \mathrm{~T} 06: 13: 48$ \\
\hline 176.05 & -19.37 & 12 & 6.9 & $2017-01-03 \mathrm{~T} 21: 52: 30$ \\
\hline & & & & \\
\hline
\end{tabular}




\begin{tabular}{|c|c|c|c|c|}
\hline-73.94 & -43.41 & 38 & 7.6 & $2016-12-25 \mathrm{~T} 14: 22: 27$ \\
\hline 153.52 & -4.5 & 95 & 7.9 & $2016-12-17 \mathrm{~T} 10: 51: 10$ \\
\hline 161.13 & -10.75 & 20 & 6.9 & $2016-12-09 \mathrm{~T} 19: 10: 06$ \\
\hline 161.33 & -10.68 & 40 & 7.8 & $2016-12-08 \mathrm{~T} 17: 38: 46$ \\
\hline-88.9 & 11.91 & 10 & 6.9 & $2016-11-24 \mathrm{~T} 18: 43: 47$ \\
\hline 173.05 & -42.74 & 15 & 7.8 & $2016-11-13 \mathrm{~T} 11: 02: 56$ \\
\hline 148.89 & -6 & 42 & 6.8 & $2016-10-17 \mathrm{~T} 06: 14: 58$ \\
\hline-178.24 & -19.78 & 596 & 6.9 & $2016-09-24 \mathrm{~T} 21: 28: 41$ \\
\hline 179.15 & -37.36 & 19 & 7 & $2016-09-01 \mathrm{~T} 16: 37: 57$ \\
\hline 152.79 & -3.69 & 476 & 6.8 & $2016-08-31 \mathrm{~T} 03: 11: 34$ \\
\hline-17.83 & -0.05 & 10 & 7.1 & $2016-08-29 \mathrm{~T} 04: 29: 57$ \\
\hline 94.57 & 20.92 & 82 & 6.8 & $2016-08-24 \mathrm{~T} 10: 34: 54$ \\
\hline-31.88 & -55.28 & 10 & 7.4 & $2016-08-19 \mathrm{~T} 07: 32: 22$ \\
\hline 173.12 & -22.48 & 16 & 7.2 & $2016-08-12 \mathrm{~T} 01: 26: 36$ \\
\hline 145.51 & 18.54 & 196 & 7.7 & $2016-07-29 \mathrm{~T} 21: 18: 24$ \\
\hline-26.93 & -56.24 & 78 & 7.2 & $2016-05-28 \mathrm{~T} 09: 46: 59$ \\
\hline-178.2 & -21.97 & 406 & 6.9 & $2016-05-28 \mathrm{~T} 05: 38: 50$ \\
\hline-79.62 & 0.49 & 30 & 6.9 & $2016-05-18 \mathrm{~T} 16: 46: 43$ \\
\hline 167.38 & -16.04 & 24 & 7 & $2016-04-28 \mathrm{~T} 19: 33: 24$ \\
\hline-79.92 & 0.38 & 21 & 7.8 & $2016-04-16 \mathrm{~T} 23: 58: 36$ \\
\hline 130.75 & 32.79 & 10 & 7 & $2016-04-15 \mathrm{~T} 16: 25: 06$ \\
\hline 94.86 & 23.09 & 136 & 6.9 & $2016-04-13 \mathrm{~T} 13: 55: 17$ \\
\hline 166.85 & -14.32 & 26 & 6.9 & $2016-04-03 \mathrm{~T} 08: 23: 52$ \\
\hline 94.33 & -4.95 & 24 & 7.8 & $2016-03-02 \mathrm{~T} 12: 49: 48$ \\
\hline 158.55 & 53.98 & 177 & 7.2 & $2016-01-30 \mathrm{~T} 03: 25: 12$ \\
\hline-153.34 & 59.62 & 126 & 7.1 & $2016-01-24 \mathrm{~T} 10: 30: 29$ \\
\hline 129.51 & -4.11 & 21 & 6.9 & $2015-12-09 \mathrm{~T} 10: 21: 48$ \\
\hline 72.78 & 38.21 & 22 & 7.2 & $2015-12-07 \mathrm{~T} 07: 50: 05$ \\
\hline 85.09 & -47.62 & 35 & 7.1 & $2015-12-04 \mathrm{~T} 22: 25: 00$ \\
\hline-71.02 & -10.06 & 621 & 7.6 & $2015-11-24 \mathrm{~T} 22: 50: 54$ \\
\hline-70.94 & -10.54 & 606 & 7.6 & $2015-11-24 \mathrm{~T} 22: 45: 38$ \\
\hline 158.42 & -8.9 & 13 & 6.8 & $2015-11-18 \mathrm{~T} 18: 31: 04$ \\
\hline-72.06 & -29.51 & 10 & 6.9 & $2015-11-11 \mathrm{~T} 02: 46: 19$ \\
\hline-72.01 & -29.51 & 12 & 6.9 & $2015-11-11 \mathrm{~T} 01: 54: 38$ \\
\hline-71.45 & -30.88 & 46 & 6.8 & $2015-11-07 \mathrm{~T} 07: 31: 43$ \\
\hline 70.37 & 36.52 & 231 & 7.5 & $2015-10-26 \mathrm{~T} 09: 09: 42$ \\
\hline 167.3 & -14.86 & 135 & 7.1 & $2015-10-20 \mathrm{~T} 21: 52: 02$ \\
\hline-71.43 & -31.56 & 28 & 7 & $2015-09-16 \mathrm{~T} 23: 18: 41$ \\
\hline & & & & \\
\hline
\end{tabular}




\begin{tabular}{|c|c|c|c|c|}
\hline-71.67 & -31.57 & 22 & 8.3 & $2015-09-16 \mathrm{~T} 22: 54: 32$ \\
\hline 138.53 & -2.63 & 48 & 7 & $2015-07-27 \mathrm{~T} 21: 41: 21$ \\
\hline-169.45 & 52.38 & 29 & 6.9 & $2015-07-27 \mathrm{~T} 04: 49: 46$ \\
\hline 165.14 & -10.4 & 11 & 7 & $2015-07-18 \mathrm{~T} 02: 27: 33$ \\
\hline-17.16 & -35.36 & 10 & 7 & $2015-06-17 \mathrm{~T} 12: 51: 32$ \\
\hline 140.49 & 27.84 & 664 & 7.8 & $2015-05-30 \mathrm{~T} 11: 23: 02$ \\
\hline-156.43 & 56.59 & 73 & 6.8 & $2015-05-29 \mathrm{~T} 07: 00: 09$ \\
\hline 163.22 & -11.11 & 10 & 6.8 & $2015-05-22 \mathrm{~T} 23: 59: 33$ \\
\hline 163.7 & -11.06 & 11 & 6.9 & $2015-05-22 \mathrm{~T} 21: 45: 19$ \\
\hline 164.17 & -10.88 & 11 & 6.8 & $2015-05-20 \mathrm{~T} 22: 48: 53$ \\
\hline 142.03 & 38.91 & 35 & 6.8 & $2015-05-12 \mathrm{~T} 21: 12: 58$ \\
\hline 86.07 & 27.81 & 15 & 7.3 & $2015-05-12 \mathrm{~T} 07: 05: 19$ \\
\hline 154.56 & -7.22 & 10 & 7.1 & $2015-05-07 \mathrm{~T} 07: 10: 19$ \\
\hline 151.88 & -5.46 & 55 & 7.5 & $2015-05-05 \mathrm{~T} 01: 44: 06$ \\
\hline 151.78 & -5.2 & 44 & 6.8 & $2015-05-01 \mathrm{~T} 08: 06: 03$ \\
\hline 152.56 & -4.73 & 41 & 7.5 & $2015-03-29 \mathrm{~T} 23: 48: 31$ \\
\hline 122.53 & -7.3 & 552 & 7 & $2015-02-27 \mathrm{~T} 13: 45: 05$ \\
\hline-31.9 & 52.65 & 17 & 7.1 & $2015-02-13 \mathrm{~T} 18: 59: 12$ \\
\hline 168.52 & -17.03 & 220 & 6.8 & $2015-01-23 \mathrm{~T} 03: 47: 27$ \\
\hline 126.58 & 1.96 & 39 & 6.8 & $2014-11-26 \mathrm{~T} 14: 33: 43$ \\
\hline 126.52 & 1.89 & 45 & 7.1 & $2014-11-15 \mathrm{~T} 02: 31: 41$ \\
\hline-177.76 & -19.69 & 434 & 7.1 & $2014-11-01 \mathrm{~T} 18: 57: 22$ \\
\hline-88.12 & 12.53 & 40 & 7.3 & $2014-10-14 \mathrm{~T} 03: 51: 34$ \\
\hline-110.81 & -32.11 & 17 & 7 & $2014-10-09 \mathrm{~T} 02: 14: 31$ \\
\hline-73.57 & -14.6 & 101 & 6.8 & $2014-08-24 \mathrm{~T} 23: 21: 45$ \\
\hline 146.17 & 0.83 & 13 & 6.9 & $2014-08-03 \mathrm{~T} 00: 22: 03$ \\
\hline-178.4 & -19.8 & 615 & 6.9 & $2014-07-21 \mathrm{~T} 14: 54: 41$ \\
\hline-92.46 & 14.72 & 53 & 6.9 & $2014-07-07 \mathrm{~T} 11: 23: 54$ \\
\hline 178.74 & 51.85 & 109 & 7.9 & $2014-06-23 \mathrm{~T} 20: 53: 09$ \\
\hline-177.72 & -29.98 & 20 & 6.9 & $2014-06-23 \mathrm{~T} 19: 19: 15$ \\
\hline 155.02 & -6.75 & 43 & 7.5 & $2014-04-19 \mathrm{~T} 13: 28: 00$ \\
\hline-100.97 & 17.4 & 24 & 7.2 & $2014-04-18 \mathrm{~T} 14: 27: 24$ \\
\hline 8.72 & -53.5 & 11 & 6.8 & $2014-04-15 \mathrm{~T} 03: 57: 01$ \\
\hline 162.05 & -11.46 & 39 & 7.4 & $2014-04-13 \mathrm{~T} 12: 36: 19$ \\
\hline 162.15 & -11.27 & 23 & 7.6 & $2014-04-12 \mathrm{~T} 20: 14: 39$ \\
\hline 155.05 & -6.59 & 61 & 7.1 & $2014-04-11 \mathrm{~T} 07: 07: 23$ \\
\hline-70.49 & -20.57 & 22 & 7.7 & $2014-04-03 \mathrm{~T} 02: 43: 13$ \\
\hline-70.94 & -19.89 & 28 & 6.9 & $2014-04-01 \mathrm{~T} 23: 57: 58$ \\
\hline & & & & \\
\hline
\end{tabular}




\begin{tabular}{|c|c|c|c|c|}
\hline-70.77 & -19.61 & 25 & 8.2 & $2014-04-01 \mathrm{~T} 23: 46: 47$ \\
\hline-125.13 & 40.83 & 16 & 6.8 & $2014-03-10 \mathrm{~T} 05: 18: 13$ \\
\hline 82.59 & 35.91 & 10 & 6.9 & $2014-02-12 \mathrm{~T} 09: 19: 49$ \\
\hline-55 & -53.95 & 12 & 7 & $2013-11-25 \mathrm{~T} 06: 27: 33$ \\
\hline-46.4 & -60.27 & 10 & 7.7 & $2013-11-17 \mathrm{~T} 09: 04: 55$ \\
\hline 144.66 & 37.16 & 35 & 7.1 & $2013-10-25 \mathrm{~T} 17: 10: 19$ \\
\hline 154.93 & -6.45 & 35 & 6.8 & $2013-10-16 \mathrm{~T} 10: 30: 58$ \\
\hline 124.12 & 9.88 & 19 & 7.1 & $2013-10-15 \mathrm{~T} 00: 12: 32$ \\
\hline 65.5 & 27.18 & 12 & 6.8 & $2013-09-28 \mathrm{~T} 07: 34: 06$ \\
\hline-74.51 & -15.84 & 40 & 7.1 & $2013-09-25 \mathrm{~T} 16: 42: 43$ \\
\hline 65.5 & 26.95 & 15 & 7.7 & $2013-09-24 \mathrm{~T} 11: 29: 47$ \\
\hline-175.23 & 51.54 & 29 & 7 & $2013-08-30 \mathrm{~T} 16: 25: 02$ \\
\hline-25.07 & -60.86 & 11 & 7.3 & $2013-07-15 \mathrm{~T} 14: 03: 39$ \\
\hline 153.93 & -3.92 & 386 & 7.3 & $2013-07-07 \mathrm{~T} 18: 35: 30$ \\
\hline 153.22 & 54.89 & 598 & 8.3 & $2013-05-24 \mathrm{~T} 05: 44: 48$ \\
\hline-177.23 & -23.01 & 174 & 7.4 & $2013-05-23 \mathrm{~T} 17: 19: 04$ \\
\hline 145.29 & 18.73 & 602 & 6.8 & $2013-05-14 \mathrm{~T} 00: 32: 25$ \\
\hline 150.79 & 46.22 & 110 & 7.2 & $2013-04-19 \mathrm{~T} 03: 05: 52$ \\
\hline 62 & 28.03 & 80 & 7.7 & $2013-04-16 \mathrm{~T} 10: 44: 20$ \\
\hline 138.48 & -3.52 & 66 & 7 & $2013-04-06 \mathrm{~T} 04: 42: 35$ \\
\hline 157.28 & 50.95 & 41 & 6.9 & $2013-02-28 \mathrm{~T} 14: 05: 50$ \\
\hline-77.39 & 1.14 & 145 & 6.9 & $2013-02-09 \mathrm{~T} 14: 16: 07$ \\
\hline 166.02 & -10.93 & 21 & 7.1 & $2013-02-08 \mathrm{~T} 15: 26: 38$ \\
\hline 165.97 & -10.84 & 12 & 6.8 & $2013-02-08 \mathrm{~T} 11: 12: 11$ \\
\hline 164.88 & -11.18 & 10 & 7.1 & $2013-02-06 \mathrm{~T} 01: 23: 19$ \\
\hline 165.11 & -10.8 & 24 & 8 & $2013-02-06 \mathrm{~T} 01: 12: 25$ \\
\hline 143.09 & 42.77 & 107 & 6.9 & $2013-02-02 \mathrm{~T} 14: 17: 35$ \\
\hline-70.65 & -28.09 & 45 & 6.8 & $2013-01-30 \mathrm{~T} 20: 15: 43$ \\
\hline 129.82 & -6.53 & 155 & 7.1 & $2012-12-10 \mathrm{~T} 16: 53: 08$ \\
\hline 143.95 & 37.89 & 31 & 7.3 & $2012-12-07 \mathrm{~T} 08: 18: 23$ \\
\hline 95.89 & 23 & 14 & 6.8 & $2012-11-11 \mathrm{~T} 01: 12: 38$ \\
\hline-91.89 & 13.99 & 24 & 7.4 & $2012-11-07 \mathrm{~T} 16: 35: 46$ \\
\hline-132.1 & 52.79 & 14 & 7.8 & $2012-10-28 \mathrm{~T} 03: 04: 08$ \\
\hline-76.36 & 1.93 & 170 & 7.3 & $2012-09-30 \mathrm{~T} 16: 31: 35$ \\
\hline-85.31 & 10.09 & 35 & 7.6 & $2012-09-05 \mathrm{~T} 14: 42: 07$ \\
\hline 126.64 & 10.81 & 28 & 7.6 & $2012-08-31 \mathrm{~T} 12: 47: 33$ \\
\hline-10.61 & 71.44 & 14 & 6.8 & $2012-08-30 \mathrm{~T} 13: 43: 25$ \\
\hline-88.59 & 12.14 & 28 & 7.3 & $2012-08-27 \mathrm{~T} 04: 37: 19$ \\
\hline & & & & \\
\hline
\end{tabular}




\begin{tabular}{|c|c|c|c|c|}
\hline 145.06 & 49.8 & 583 & 7.7 & $2012-08-14 \mathrm{~T} 02: 59: 38$ \\
\hline 147.12 & -5.46 & 198 & 6.8 & $2012-04-17 \mathrm{~T} 07: 13: 49$ \\
\hline-113.1 & 28.7 & 13 & 7 & $2012-04-12 \mathrm{~T} 07: 15: 48$ \\
\hline 92.46 & 0.8 & 25 & 8.2 & $2012-04-11 \mathrm{~T} 10: 43: 10$ \\
\hline 93.06 & 2.33 & 20 & 8.6 & $2012-04-11 \mathrm{~T} 08: 38: 36$ \\
\hline-72.22 & -35.2 & 41 & 7.1 & $2012-03-25 \mathrm{~T} 22: 37: 06$ \\
\hline-98.23 & 16.49 & 20 & 7.4 & $2012-03-20 \mathrm{~T} 18: 02: 47$ \\
\hline 144.94 & 40.89 & 12 & 6.9 & $2012-03-14 \mathrm{~T} 09: 08: 35$ \\
\hline 167.13 & -17.83 & 23 & 7.1 & $2012-02-02 \mathrm{~T} 13: 34: 40$ \\
\hline 93.21 & 2.43 & 19 & 7.2 & $2012-01-10 \mathrm{~T} 18: 36: 59$ \\
\hline 138.07 & 31.46 & 365 & 6.8 & $2012-01-01 \mathrm{~T} 05: 27: 55$ \\
\hline 146.81 & -7.55 & 135 & 7.1 & $2011-12-14 \mathrm{~T} 05: 04: 58$ \\
\hline 125.62 & 27.32 & 225 & 6.9 & $2011-11-08 \mathrm{~T} 02: 59: 08$ \\
\hline-75.97 & -14.44 & 24 & 6.9 & $2011-10-28 \mathrm{~T} 18: 54: 34$ \\
\hline 43.51 & 38.72 & 18 & 7.1 & $2011-10-23 \mathrm{~T} 10: 41: 23$ \\
\hline-176.24 & -28.99 & 33 & 7.4 & $2011-10-21 \mathrm{~T} 17: 57: 16$ \\
\hline 88.16 & 27.73 & 50 & 6.9 & $2011-09-18 \mathrm{~T} 12: 40: 51$ \\
\hline-179.53 & -21.61 & 645 & 7.3 & $2011-09-15 \mathrm{~T} 19: 31: 04$ \\
\hline 169.72 & -20.67 & 185 & 7 & $2011-09-03 \mathrm{~T} 22: 55: 40$ \\
\hline-171.71 & 52.17 & 32 & 6.9 & $2011-09-02 \mathrm{~T} 10: 55: 53$ \\
\hline 126.75 & -6.36 & 470 & 6.9 & $2011-08-30 \mathrm{~T} 06: 57: 41$ \\
\hline-74.53 & -7.64 & 147 & 7 & $2011-08-24 \mathrm{~T} 17: 46: 11$ \\
\hline 168.22 & -18.31 & 28 & 7.1 & $2011-08-20 \mathrm{~T} 18: 19: 23$ \\
\hline 168.14 & -18.36 & 32 & 7.2 & $2011-08-20 \mathrm{~T} 16: 55: 02$ \\
\hline 143.26 & 38.03 & 23 & 7 & $2011-07-10 \mathrm{~T} 00: 57: 10$ \\
\hline-176.34 & -29.54 & 17 & 7.6 & $2011-07-06 \mathrm{~T} 19: 03: 18$ \\
\hline-171.84 & 52.05 & 52 & 7.3 & $2011-06-24 \mathrm{~T} 03: 09: 39$ \\
\hline 168.23 & -20.24 & 11 & 6.8 & $2011-05-10 \mathrm{~T} 08: 55: 08$ \\
\hline 161.2 & -10.38 & 79 & 6.8 & $2011-04-23 \mathrm{~T} 04: 16: 54$ \\
\hline 141.59 & 38.28 & 42 & 7.1 & $2011-04-07 \mathrm{~T} 14: 32: 43$ \\
\hline 144.59 & 38.06 & 19 & 7.7 & $2011-03-11 \mathrm{~T} 06: 25: 50$ \\
\hline 141.11 & 36.28 & 43 & 7.9 & $2011-03-11 \mathrm{~T} 06: 15: 40$ \\
\hline 142.37 & 38.3 & 29 & 9.1 & $2011-03-11 \mathrm{~T} 05: 46: 24$ \\
\hline 142.84 & 38.44 & 32 & 7.3 & $2011-03-09 \mathrm{~T} 02: 45: 20$ \\
\hline-72.96 & -36.42 & 26 & 6.9 & $2011-02-11 \mathrm{~T} 20: 05: 30$ \\
\hline 63.95 & 28.78 & 68 & 7.2 & $2011-01-18 \mathrm{~T} 20: 23: 23$ \\
\hline-73.33 & -38.35 & 24 & 7.2 & $2011-01-02 \mathrm{~T} 20: 20: 17$ \\
\hline-63.14 & -26.8 & 577 & 7 & $2011-01-01 \mathrm{~T} 09: 56: 58$ \\
\hline & & & & \\
\hline
\end{tabular}




\begin{tabular}{|c|c|c|c|c|}
\hline 167.95 & -19.7 & 16 & 7.3 & 2010-12-25T13:16:37 \\
\hline 143.7 & 26.9 & 14 & 7.4 & 2010-12-21T17:19:40 \\
\hline 139.19 & 28.35 & 470 & 6.8 & 2010-11-30T03:24:40 \\
\hline 100.08 & -3.49 & 20 & 7.8 & 2010-10-25T14:42:22 \\
\hline 133.76 & -4.96 & 26 & 7 & 2010-09-29T17:11:25 \\
\hline 171.83 & -43.52 & 12 & 7 & 2010-09-03Т16:35:47 \\
\hline 141.47 & 12.49 & 16 & 6.9 & 2010-08-13T21:19:34 \\
\hline-77.31 & -1.27 & 207 & 7.1 & 2010-08-12T11:54:15 \\
\hline 168.07 & -17.54 & 25 & 7.3 & 2010-08-10T05:23:44 \\
\hline 150.76 & -5.75 & 44 & 7 & 2010-08-04T22:01:43 \\
\hline 123.26 & 6.78 & 641 & 7.5 & 2010-07-23Т23:15:10 \\
\hline 123.48 & 6.5 & 578 & 7.6 & 2010-07-23T22:51:11 \\
\hline 123.41 & 6.72 & 607 & 7.3 & 2010-07-23T22:08:11 \\
\hline 150.59 & -5.93 & 35 & 7.3 & 2010-07-18T13:34:59 \\
\hline 150.43 & -5.97 & 28 & 6.9 & 2010-07-18T13:04:09 \\
\hline 136.54 & -2.17 & 18 & 7 & 2010-06-16T03:16:27 \\
\hline 91.94 & 7.88 & 35 & 7.5 & 2010-06-12T19:26:50 \\
\hline 166.64 & -13.7 & 31 & 7.2 & 2010-05-27T17:14:46 \\
\hline 96.02 & 3.75 & 38 & 7.2 & 2010-05-09T05:59:41 \\
\hline 96.55 & 33.16 & 17 & 6.9 & 2010-04-13T23:49:38 \\
\hline 161.12 & -10.88 & 21 & 6.9 & 2010-04-11T09:40:25 \\
\hline 97.05 & 2.38 & 31 & 7.8 & 2010-04-06T22:15:01 \\
\hline-71.8 & -34.33 & 18 & 7 & 2010-03-11T14:55:27 \\
\hline-71.89 & -34.29 & 11 & 6.9 & 2010-03-11T14:39:43 \\
\hline 100.99 & -3.76 & 26 & 6.8 & 2010-03-05T16:07:00 \\
\hline-75.05 & -37.77 & 35 & 7.4 & 2010-02-27T08:01:23 \\
\hline-72.9 & -36.12 & 23 & 8.8 & 2010-02-27T06:34:11 \\
\hline 128.43 & 25.93 & 25 & 7 & $2010-02-26 \mathrm{~T} 20: 31: 26$ \\
\hline 130.7 & 42.59 & 578 & 6.9 & 2010-02-18T01:13:19 \\
\hline-72.57 & 18.44 & 13 & 7 & $2010-01-12 \mathrm{~T} 21: 53: 10$ \\
\hline 157.55 & -9.02 & 15 & 6.8 & 2010-01-05T12:15:32 \\
\hline-14.7 & -58.17 & 13 & 6.8 & 2010-01-05T04:55:39 \\
\hline 157.35 & -8.78 & 10 & 7.1 & 2010-01-03T22:36:25 \\
\hline
\end{tabular}




\section{Reference}

Auld, B. A. (1990). Acoustic fields and waves in solids. Vol. 1 Vol. 1. Malabar: Krieger Publishing Company.

Chapman, C. H. (1981). Generalized Radon transforms and slant stacks. Geophysical Journal International, 66(2), 445-453. https://doi.org/10.1111/j.1365-246X.1981.tb05966.x

Kennett, B. L. N., Engdahl, E. R., \& Buland, R. (1995). Constraints on seismic velocities in the Earth from traveltimes. Geophysical Journal International, 122(1), 108-124. https://doi.org/10.1111/j.1365-246X.1995.tb03540.x

Lin, J.-F., Mao, Z., Yavaş, H., Zhao, J., \& Dubrovinsky, L. (2010). Shear wave anisotropy of textured hcp-Fe in the Earth's inner core. Earth and Planetary Science Letters, 298(3), 361-366. https://doi.org/10.1016/j.eps1.2010.08.006

Mao, W. L., Struzhkin, V. V., Baron, A. Q. R., Tsutsui, S., Tommaseo, C. E., Wenk, H.-R., et al. (2008). Experimental determination of the elasticity of iron at high pressure. Journal of Geophysical Research: Solid Earth, 113(B9). https://doi.org/10.1029/2007JB005229

Mattesini, M., Belonoshko, A. B., Buforn, E., Ramírez, M., Simak, S. I., Udías, A., et al. (2010). Hemispherical anisotropic patterns of the Earth's inner core. Proceedings of the National Academy of Sciences, 107(21), 9507-9512. https://doi.org/10.1073/pnas.1004856107

Phạm, T.-S., Tkalčić, H., Sambridge, M., \& Kennett, B. L. N. (2018). Earth’s Correlation Wavefield: Late Coda Correlation. Geophysical Research Letters, 45(7), 3035-3042. https://doi.org/10.1002/2018GL077244

Romanowicz, B., Cao, A., Godwal, B., Wenk, R., Ventosa, S., \& Jeanloz, R. (2016). Seismic anisotropy in the Earth’s innermost inner core: Testing structural models against mineral physics predictions. Geophysical Research Letters, 43(1), 93-100. https://doi.org/10.1002/2015GL066734

Shearer, P. M. (1994). Constraints on inner core anisotropy from PKP(DF) travel times. Journal of Geophysical Research: Solid Earth, 99(B10), 19647-19659. https://doi.org/10.1029/94JB01470

Song, X. (1997). Anisotropy of the Earth's inner core. Reviews of Geophysics, 35(3), 297-313. https://doi.org/10.1029/97RG01285

Song, X., \& Helmberger, D. V. (1995). Depth dependence of anisotropy of Earth's inner core. Journal of Geophysical Research: Solid Earth, 100(B6), 9805-9816. https://doi.org/10.1029/95JB00244

Vočadlo, L., Dobson, D. P., \& Wood, I. G. (2009). Ab initio calculations of the elasticity of hep-Fe as a function of temperature at inner-core pressure. Earth and Planetary Science Letters, 288(3), 534-538. https://doi.org/10.1016/j.eps1.2009.10.015 
manuscript submitted to Geophysical Research Letters

828 Wang, S., \& Tkalčić, H. (2020). Seismic event coda-correlation's formation: implications for global seismology. Geophysical 829 Journal International, 222(2), 1283-1294. https://doi.org/10.1093/gji/ggaa259

830

831

832

833 